\title{
Relational Political Contribution under Common Agency*
}

\author{
Akifumi Ishihara $^{\dagger}$
}

July 27, 2009

\begin{abstract}
A model of political contribution of dynamic common agency where state-contingent agreements must be self-enforced is considered. Two issues are mainly examined; punishment strategy on deviation and the limit and scope of implicit agreements. The punishment strategy on the principals takes a form of two-phase scheme in general and, more specifically, it is either an "Exclusion-type", on which the bad decision on the deviator is keep to be chosen over time, or a "Sanction-type" which is indeed same as the so-called stick-and-carrot strategy. Furthermore we characterize the payoff set of the equilibria on which the same decision is chosen by the agent through implicit agreements and compare it with those in the standard static menu auction model. One of the main implications is that there could be an equilibrium outcome which cannot be supported on our model even the discount factor is close to one.
\end{abstract}

Keyword: Common Agency, Relational Contract, Lobbying

JEL classification: D86, C73, D72, L14

\section{Introduction}

Because of intention to influence the political decision, monetary transfer between politicians and lobbyists from interest groups is observed in many political processes, as a form of campaign contributions, illegal bribes, and so on. The contributors have their own interest or preference for the decision, which could conflict with each other. Thus they often compete strategically to obtain the desirable decision through many forms of transfer.

Common agency model is a widely spreading framework to analyze it. The model contains an agent, who is a decision maker, and two or more principals, each of whom offers a contract contingent on the agent's decision. The seminal paper by Bernheim and Whinston (1986b, hereafter

\footnotetext{
${ }^{*}$ I am greatly indebted to Andrea Prat for kindly supervising activity. I am benefited from Leonardo Felli, Hideshi Itoh, Michele Piccione, and Yuichi Yamamoto for comments and discussions. I wish to thank participants at CTW Summer Conference at Hokkaido university, and seminar at Hitotsubashi university and LSE. All the errors are responsible for

${ }^{\dagger}$ Department of Economics and STICERD, London School of Economics and Political Science. E-mail:
} me. A.ishihara@lse.ac.uk 
$\mathrm{BW})^{1)}$ consider menu auction to be common agency such that the bidders offer a payment plan contingent on the auctioneer's decision on the allocation of the goods. Since then, this menu auction model has been applied to the analysis of buying influence through political contribution from lobbyists and a politician to investigate many issues in political economy.

The implicit assumption behind the analysis by menu auction model is long term relationship between the politician and the lobbyists while the analyzed model typically appears as one shot game. ${ }^{2)}$ In BW, a payment plan offered by the principals is regarded as an explicit contract so that it would be enforced by the court or another third party after the agent makes an decision. However, to assume that the lobbyist can offer such an explicit contract contingent on the political decision to the politician is far from reality. Then, at least in the context of politics, such a compensation contract must be just an implicit agreement and hence we need another justification for the principal's ability to commit to the implicit agreement. One of them is the repeated relationship which creates the serious punishment if the agreement is reneged on.

Whereas there are a certain amount of documents describing long-term interaction among politicians and leaders of interest groups involved in political decision process, it still does not seem to be enough to justify the commitment assumption. Some empirical evidences support this view. For example, McCarty and Rothenberg (1996) empirically find that in the U.S. the credible commitment of campaign contribution by Political Action Committee is weak. Another empirical evidence is from Snyder (1992) who reports that (i) campaign contribution to young representatives in the U.S. House is larger than old representatives and (ii) representatives running in small states received more contribution than in large states, implying that the contributors seriously pay attention to how long their relation is going on. While it is still umbiguous whether we can reject the assumption of quite patient political players, these evidences suggest that it seems to be questionable that the binding contract can be an approximation of the implicit agreement and then

\footnotetext{
${ }^{1)}$ Bernheim and Whinston (1986a), the title of which is exactly "Common Agency", extends to the model with hidden decision by the agent while Bernheim and Whinston (1986b) do not include any asymmetric information. However we usually call the model without asymmetric information "common agency" as well as with hidden decision.

${ }^{2)}$ Some authors explicitly mention this notion. For example, see Grossman and Helpman (2001, p. 228).
} 
it is required to analyze explicitly the situation where the involved people must make a credible punishment by themselves.

The present paper tries the formal analysis of the political contribution which takes a form of implicit agreements and then must be self-enforced. The literature on the theory of relational contracts $^{3)}$ has already studied self-enforced contract in dynamic situations by applying the framework of the repeated game. Recently, tractable frameworks of relational contracts have been established, especially by MacLeod and Malcomson (1989) and Levin (2003). Basically, the bottom line of our model is based on their models. Our model departs from them in two features. First, while the existing model analyzes the bilateral relations between a single principal and a single agent, our model allows multi-principal situations. Second, it is assumed that the players have no outside option which is a more realistic assumption in the political process, the reason of which is discussed later.

Specifically, our model is summarized by the following features; (i) the model is an infinitely repeated common agency game with perfect information, (ii) the principals cannot offer explicit binding contracts at all, and (iii) all the players have no outside option to exit from the relation. The analysis essentially consists of two parts. First, the punishment strategy on deviation is examined. The punishment on a deviator is driving force to sustain relational contracts and then it gives us a micro foundation for the mechanism to enforce the political contribution. Second, we fully characterize the stationary equilibrium payoff set in the sense that the agents chooses the same decision repeatedly. It exhibits the scope of implicit contracts and an implication from the results shows that, contrary to the theoretical conventional wisdom, an equilibrium in the standard menu auction model might not be supported on implicit contracts no matter how patient the players are.

In general, the more harsh the punishment is, the more credible the implicit agreement is. Then solving the minimization problem gives the minimum equilibrium payoff which is targeted when the punishment starts. Moreover it is illustrated that the punishment strategy is drastically

\footnotetext{
${ }^{3)}$ It is sometimes also called as "implicit contracts" as opposed to the explicit contract.
} 
different from the existing models of relational contracts which is due to the lack of outside options in political contribution model. In the existing model such as MacLeod and Malcomson (1989) and Levin (2003), while the players are not allowed to write a binding contract contingent on the state variable, there is an explicit contract to construct the relation (e.g. the employer employs the employee or the seller and the buyers agree to a future contract) and if the players do not participate in the contract, they obtain the (constant) reservation utility from the outside option. Under some mild assumptions, it assures that the optimal punishment on a deviator from the equilibrium behaviour would be without loss of generality simplified to termination of the relation forever. Meanwhile, in political situations, the effect by the political decision is essentially independent from whether an agreement is achieved between the lobbyist and the politician. For instance, in case of protection for sale, the industry group always cares about imports and/or exports from foreign countries, which is influenced by the tariff policy. However the event to access the politician and to make an agreement itself does not affect the trade quantity at all. This independence is similarly applicable to the politician. It implies that that it is reasonable to presume that the players cannot escape from the agent's decision, implying that they do not have an outside option. ${ }^{4)}$

The natural benchmark for the optimal punishment is the repetition of the stage game equilibrium; the principals pay nothing and the agent chooses the decision he prefers the most. Actually it is the optimal punishment on the deviation by the agent. However, it need not be on the deviation by the principal. For example, if the decision is undesirable for the deviating principal but desirable for the other principals, the exclusive behaviour such that this decision is chosen repeatedly can be the punishment strategy. However, even if the undesirable decision for the deviating principal is also undesirable for all the players, the harsh payoff can be achieved by the following one-shot sanction similar to the so-called "stick and carrot" punishment first analyzed

\footnotetext{
${ }^{4)}$ It is said that lobbyists also influence the politician through giving information. (see Grossman and Helpman (2001, Part 2).) If the information transmission happens only when the lobbyist meets the politician and the policy can be changed only if the politician gets information from the lobbyists, a situation of which is a drastic amendment, the event of meeting might make an outside option. Although we agree that the role of information transmission by lobbyists is an important issue, it is abstracted here.
} 
by Abreu (1986) in the context of Cournot oligopoly. Suppose that there is another decision which is relatively desirable for all of the players, including the deviating principal. Now think of the following public announcement; "we will take the undesirable decision as a sanction on the deviator. But once the deviator pays some fine to the agent, we will stop the sanction and go back to the desirable decision." If the deviator follows to pay the fine, she voluntarily incurs the additional cost by paying the fine as well as the cost from the undesirable decision. However, once she pays the fine, the undesirable decision is immediately going to stop and all the players, including the deviator, would be happy. Thus all the players can put up with the undesirable decision if it happens only once. Thus, by setting an appropriate fine, the deviating principal is willing to pay the fine. Then this punishment can be credible. The punishment strategy would be without loss of generality either a repeated punishment called an Exclusion-type or a stick-and-carrot punishment called a Sanction-type.

The other issue to be examined is decision-stationary equilibria, on which the agent chooses the same decision repeatedly over time. We will show that when investigating decision-stationary equilibria, we can restrict the principal's strategy within the stationary class in the sense that the principals offer the same rewarding schedule repeatedly at the equilibrium path. Furthermore, we derive the necessary and sufficient condition for the existence of decision-stationary equilibria. These results are similar to the previous literature of relational contracts and not so surprising. Our main contribution is a full characterization of the set of the decision-stationary equilibrium payoffs, which can then be compared with the equilibrium payoffs in the static menu auction model of BW. In particular, we will derive the necessary and sufficient condition under which the payoff of the equilibrium in the corresponding static menu auction can be supported by the equilibria in the corresponding dynamic political contribution model. ${ }^{5)}$ Not surprisingly a static equilibrium payoff is more likely to be an equilibrium payoff in a dynamic relational common agency if the discount factor is higher. In particular, we see the existence of the upper bound of the

\footnotetext{
${ }^{5)}$ In what follows, we refer "menu auction" as environments where binding contracts are allowed and "political contribution" as those without binding contracts allowed.
} 
credible relational payment and a static equilibrium payoff is possible to realize when the payment on the static equilibrium is below the upper bound. It is not difficult to see that the upper bound is increasing in the discount factor, implying that a static equilibrium payoff is more likely to appear on the relational equilibrium when the players are patient. However, an interesting result is that there could be a static equilibrium payoff which cannot be an equilibrium payoff in the relational common agency even if the discount factor is almost close to 1.

Roughly speaking, this interesting case appears when there are more than two principals each of whom is faced with a threat of being isolated from the other players by the agent's decision. In the static menu auction, because there is no cost enforcing the contract, all the players except for the principal threatened to be isolated are willing to agree to such an isolating decision, which allows the agent to exploit the threatened principal. If there are two principals being threatened, at least one of them would be exploited in a way of a positive amount of transfer. However, in order to exploit the principal via monetary transfer only by implicit agreements, the credible severe punishment is required and the effect of the punishment is always discounted because the punishment is invoked only after the deviation is observed and then it must be delayed. Hence, in the extreme case, no matter how much the players are patient, the punishment available in the relational case is not enough to exploit the principal through a monetary transfer. Then the exploitation from more than one principal would be impossible by implicit contracts whereas it is possible in the static menu auction.

This result means that, given any discount factor fixed, the equilibrium payoff derived by the static analysis is uniformly bounded from the the set of the relational equilibrium payoffs, implying that the equilibrium obtained in static menu auctions crucially relies on the assumption that the principals can fully commit their offer without any endogenous mechanism. Thus it would suggest that the analysis of political contribution by the static menu auction would be collapsed in the above case or an additional justification other than the repeated relationship for being possible to commit is required. 
More generally, the principals are always faced with the limit of the credible amount to pay to the agent when the agreement is just implicit and it is the main reason for which the equilibrium outcome in the static model with commitment ability often collapses by implicit agreements. This observation suggests that introduction the upper bound on the feasible contracts in static menu auction models would be a legitimate but still tractable extension in the context of political economy.

The rest of this paper is organized as follows. The next section reviews the related literature. The setting of the model and the general way to analyze it is described in section 3 . The derivation of the equilibria is demonstrated from section 4 to 6 . In section 4 , the preliminary result of decisionstationary equilibria is offered. In section 5, the characterization of the punishment strategy is illustrated. These results are combined in section 6 to characterize the set of decision-stationary equilibrium payoffs. Section 7 compares it with that of the static menu auction and shows the main result. Finally section 8 concludes. The proofs are in the appendix.

\section{Related Literature}

In this section, the works related to the present paper from theoretical aspects are discussed.

As mentioned in section 1, the first step to static common agency is offered by BW. Using the model with perfect information, they characterize the equilibria and propose a concept of truthful equilibrium, which is a refined equilibrium and has Pareto efficient and coalition-proof property. Bernheim and Whinston (1986a), Dixit et al. (1997), Prat and Rustichini (2003) generalize to hidden decision, non-quasilinear utility in transfer, and multiple agents with multiple principals, respectively. Bergemann and Välimäki (2003) investigate the conditions of the existence and uniqueness of a truthful equilibrium. They also study (both finitely and infinitely) repeated common agency. In their model, however, state-contingent contracts within one period can be

\footnotetext{
${ }^{6}$ While the framework by Dixit et al. (1997) allows the environment where the principal's payment contract is restricted by the upper bound, they do not obtain the positive result implied by the upper bound. To the best of our knowledge, the solo study along this line is by Campante and Ferreira (2007). See the next section.
} 
enforced by the third party. Their interest is a characterization of Markov perfect equilibria (in the sense of Maskin and Tirole (2001)) in the stochastic game, where there is a payoff-relevant state variable which follows the Markov process. ${ }^{7)}$

BW's framework of menu auction is applied to the several political situation with lobbyists who offer monetary transfer as an incentive device. The partial list of the papers along this line is as follows; Grossman and Helpman (1994) for tariff policy, Grossman and Helpman (1996) for campaign advertising in election, Fredriksson (1997) for pollution tax policy, and Besley and Coate (2001) for representative democracy. ${ }^{8)}$ Campante and Ferreira (2007) is closely related to our model in the sense that they consider the effect of the lack of the ability to commit to the payment schedule as well as our model. However the environment imagined is different from ours. They focus on lobbyists and the government in production economy and assume that resource availability by production technology makes the credible amount of the payment limited while decision-contingent contracts are still (at least partially) possible. ${ }^{9)}$ Their main result shows inefficiency via lobbying activity due to the the lack of credibility. By contrast, we investigate the environment where the principals do not have any ability to commit to their own rewarding schedule and we study the scope of lobbying activity only by self-enforced agreements. Our result would be complementary in the sense that we provide a kind of micro foundation for limiting the credible contribution.

Self-enforced contracts have also been studied by many researchers. Particularly, the definitive framework is developed by MacLeod and Malcomson (1989) and Levin (2003) in the context of labour contract, who establish the legitimate approach by stationary relational contracts. ${ }^{10)}$ Furthermore self-enforced contracts with multiple parties are studied by Levin (2002), Kvaløy and Olsen (2006), and Rayo (2007). However all of them consider the multi-agent situation and to the best of our knowledge, no paper studies self-enforced contracts in multi-principal situation.

\footnotetext{
${ }^{7)}$ The recent developed theory of common agency is excellently surveyed by Martimort (2006).

${ }^{8)}$ See also Grossman and Helpman (2001, Part 3).

${ }^{9}$ They justify it by imagining that both the government and the lobbyists take actions simultaneously.

${ }^{10)}$ Relational contract is also studied in the theory of organization. See Baker et al. (2002), for example.
} 


\section{The Model}

\subsection{Environment}

There are $N$ principals $^{11)}$ (those are females, called $1,2, \ldots$, and $N$, respectively) and an agent (who is a male and sometimes called player 0 ), all of whom will live at infinite periods $t=0,1, \ldots$

- Denote $\mathcal{N}:=\{1,2, \ldots, N\}$ as the set of the principals. At each period $t$, the players play the following two stage game;

- stage 1: the agent chooses a decision $a_{t}$ from the decision set $\mathcal{A}$,

- stage 2: given $a_{t}$, each principal $j$ simultaneously and noncooperatively pays $b_{t}^{j} \in \mathbf{R}_{+}$to the agent.

It is assumed that at the beginning of each period $t$, all of the past decisions and payments $\left\{a_{\tau},\left\{b_{\tau}^{j}\right\}_{j=1}^{N}\right\}_{\tau=0}^{t-1}$ are observable for all the players.

When the agent chooses $a_{t}$ at period $t$, each player $i \in \mathcal{N} \cup\{0\}$ gets the one-shot benefit $v^{i}\left(a_{t}\right) \in \mathbf{R}$. Thus principal $j^{\prime}$ s one-shot net payoff is $v^{j}\left(a_{t}\right)-b_{t}^{j}$ and the agent's is $v^{0}\left(a_{t}\right)+B_{t}$ where $B_{t}:=\sum_{k=1}^{N} b_{t}^{k}$. The purpose of each player in the entire game is to maximize the average discounted sum of the her/his payoff with common discount factor $\delta \in[0,1)$, that is, $(1-\delta) \sum_{\tau=0}^{\infty} \delta^{\tau}\left[v^{j}\left(a_{\tau}\right)-b_{\tau}^{j}\right]$ for principal $j$ and $(1-\delta) \sum_{\tau=0}^{\infty} \delta^{\tau}\left[v^{0}\left(a_{\tau}\right)+B_{\tau}\right]$ for the agent.

We assume that $\mathcal{A}$ is a compact subset of $\mathbf{R}^{L}$ where $L$ is an arbitrary finite positive integer and $v^{i}(\cdot)$ is continuous for all $i \in \mathcal{N} \cup\{0\}$. These assumptions ensure the existence of the maximum and minimum of $v^{i}(a)$ and then the followings are well-defined; for all $i \in \mathcal{N} \cup\{0\}$,

$$
\bar{A}^{i}:=\underset{a \in \mathcal{A}}{\arg \max } v^{i}(a), \underline{A}^{i}:=\underset{a \in \mathcal{A}}{\arg \min } v^{i}(a), \bar{v}^{i}:=\max _{a \in \mathcal{A}} v^{i}(a), \underline{v}^{i}:=\min _{a \in \mathcal{A}} v^{i}(a) .
$$

We sometimes use a notation $\bar{a}^{i}$ and $\underline{a}^{i}$ as a representative element of $\bar{A}^{i}$ and $\underline{A}^{i}$ respectively. Moreover let $s(a):=\sum_{i=0}^{N} v^{i}(a)$ be the one shot total surplus and denote $A^{*}:=\arg \max _{a \in \mathcal{A}} s(a)$ and

\footnotetext{
${ }^{11)}$ Although common agency is imagined, all the results in this paper hold even when $N=1$.
} 
$s^{*}:=\max _{a \in \mathcal{A}} s(a)$. Denote $P^{j}(\delta):=\left[0, M^{j}(\delta)\right]$ where $M^{j}(\delta):=(\delta /(1-\delta))\left[\bar{v}^{j}-\underline{v}^{j}\right]$ for $j \in \mathcal{N}$. Notice that if principal $j$ pays more than $M^{j}(\delta)$ at some period, her discounted average payoff must be less than $\underline{v}_{j}$ which must be less than the payoff when she always pays nothing. Thus it is without loss of generality that we restrict principal $j^{\prime}$ s strategy space to $P^{j}(\delta)$, which ensures the principal's strategy space compact.

The strategy of the repeated game is defined by a mapping from an observed history to a decision or payment at the current period. Formally let

$$
\begin{aligned}
\mathcal{H}_{0}^{j} & =\emptyset \text { for } j \in \mathcal{N} \cup\{0\}, \mathcal{H}_{t}^{0}=\mathcal{A}^{t}, \mathcal{H}_{t}^{j}=P^{j}(\delta)^{t} \text { for } j \in \mathcal{N} \text { and } t=1,2, \ldots, \\
\mathcal{H}_{t} & :=\prod_{j=0}^{N} \mathcal{H}_{t}^{j}
\end{aligned}
$$

and define $\mathcal{H}:=\cup_{t=0}^{\infty} \mathcal{H}_{t}$ as the set of the histories for the agent. ${ }^{12)}$ The strategy ${ }^{13)}$ is given by $\sigma^{0}$ : $\mathcal{H} \rightarrow \mathcal{A}$ for the agent and $\sigma^{j}: \mathcal{H} \times \mathcal{A} \rightarrow P^{j}(\delta)$ for principal $j$. Let $\sigma:=\left(\sigma^{0}, \sigma^{1}, \ldots, \sigma^{N}\right)$ be its profile. The strategy profile generates the on-path outcome such as $\left(\left(a_{0}, a_{1}, \ldots\right),\left(b_{0}^{1}, b_{1}^{1}, \ldots\right), \ldots,\left(b_{0}^{N}, b_{1}^{N}, \ldots\right)\right)$ and then the discounted average payoffs can be computed from it. We adopt the subgame perfect Nash equilibrium (SPE) as our equilibrium concept where, for any history $h_{t} \in \mathcal{H}$ or $\left(h_{t}, a_{t}\right) \in \mathcal{H} \times \mathcal{A}$, the behavioural strategy is a Nash equilibrium, (or equivalently, it is a SPE). Denote $\Sigma^{*}$ be the set of SPE and $U^{*}:=\left\{u^{0}(\sigma), u^{1}(\sigma), \ldots, u^{N}(\sigma) \mid \sigma \in \Sigma^{*}\right\}$ be the set of SPE payoffs.

\subsection{Simple Strategy Representation}

The equilibrium analysis is substantially simplified by recursive formulation and the simple strategy introduced by Abreu (1988).

Let $\hat{\sigma}$ be an arbitrary SPE and $\sigma(i) \in \Sigma^{*}$ be a SPE which yields the lowest equilibrium payoff for player $i$, i.e. $u^{i}(\sigma(i)) \leq u^{i}(\sigma)$ for any $\sigma \in \Sigma^{*}$. Abreu (1988) calls $\sigma(i)$ the "optimal penal code" (OPC) and the existence can be shown. ${ }^{14)}$

\footnotetext{
${ }^{12)}$ Notice that $\mathcal{H}$ does not include the states just after the stage 1 ends (i.e. the agent chooses some decision).

${ }^{13)}$ We focus on the pure strategy.

${ }^{14)}$ Abreu (1988) shows it when the stage game is normal form while it is an extensive form game here. Nevertheless
} 
Now consider the following "simple strategy" profile $\sigma^{S S}(\hat{\sigma}, \sigma(0), \sigma(1), \ldots, \sigma(N))$, such that

- to follow $\hat{\sigma}$ if no player has deviated,

- to pay nothing and follow $\sigma(0)$ from next period if the agent deviates, and

- to change to $\sigma(j)$ from next period if principal $j$ deviates.

By construction of $\sigma^{S S}$, if player $i$ chooses strategy $\sigma^{S S^{i}}$ (provided that the other players follow $\sigma^{S S^{-i}}$ ), he/she follows strategy $\hat{\sigma}^{i}$ if no player has deviated from the outcome path of $\hat{\sigma}$ and immediately moves to the strategy which yields the lowest equilibrium payoff for the deviating player otherwise.

We can show the following lemma by a little modified proof of Abreu (1988, Proposition 5). ${ }^{15)}$

Lemma 1 Suppose that there exists the OPC $\sigma(i)$ for all $i \in \mathcal{N} \cup\{0\}$. Then if $\hat{\sigma} \in \Sigma^{*}, \sigma^{S S}(\hat{\sigma}, \sigma(0), \sigma(1), \ldots, \sigma(N)) \in$ $\Sigma^{*}$

The generating outcome of $\sigma^{S S}(\hat{\sigma}, \sigma(0), \sigma(1), \ldots, \sigma(N)) \in \Sigma^{*}$ is same as that of $\hat{\sigma}$. Then Lemma 1 allows us to focus on the simple strategy with the OPC to analyze the equilibrium outcome. In what follows, we will focus the simple strategy SPE unless noted explicitly. ${ }^{16)}$

Now let $\hat{\sigma}$ be an arbitrary simple strategy profile. The one-shot deviation principle implies that $\hat{\sigma}$ is a SPE if and only if

- the players have no deviation incentive at period 0 , and

- the continuation strategy profile is a SPE.

We will formally describe these conditions.

From $\hat{\sigma}$, we can construct the equilibrium path such that $\hat{a}_{0}=\hat{\sigma}^{0}(\emptyset), \hat{b}_{0}^{j}=\hat{\sigma}^{j}\left(\hat{a}_{0}\right)$, and the continuation strategy profile $\hat{\sigma}_{1}(\cdot) \equiv \hat{\sigma}\left(\hat{a}_{0}, \hat{\boldsymbol{b}}_{0}, \cdot\right)$ where $\hat{\boldsymbol{b}}_{0}:=\left(\hat{b}_{0}^{1}, \ldots, \hat{b}_{0}^{N}\right)$. First, look at the agent's deviation incentive at period 0 . If the agent follows $\hat{\sigma}^{0}$ at period 0 , the average payoff the agent

it is enough to modify his proof a little for proving the existence. The proof is omitted.

${ }^{15)}$ The formal proof is omitted.

${ }^{16)}$ Notice that the OPC is also a simple strategy such as $\sigma(j)=\sigma^{S S}(\sigma(j), \sigma(0), \sigma(1), \ldots, \sigma(N))$. 
obtains has a following recursive expression as

$$
u^{0}(\hat{\sigma})=(1-\delta)\left[\hat{B}_{0}+v^{0}\left(\hat{a}_{0}\right)\right]+\delta u^{0}\left(\hat{\sigma}_{1}\right)
$$

If he deviates to another decision $a^{\prime}$, he gains the benefit $v^{0}\left(a^{\prime}\right)$ at period 0 and, since the players start the punishment on the agent, he receives nothing and the continuation strategy profile would be $\sigma(0)$. Then the utility is $(1-\delta) v^{0}\left(a^{\prime}\right)+\delta u^{0}(\sigma(0))$. Notice that $(1-\delta) v^{0}\left(a^{\prime}\right)+\delta u^{0}(\sigma(0)) \leq(1-\delta) \bar{v}^{0}+\delta u^{0}(\sigma(0))$ for all $a^{\prime} \in \mathcal{A}$. Thus the agent does not deviate from $\hat{\sigma}$ at period 0 if and only if

$$
(1-\delta)\left[\hat{B}_{0}+v^{0}\left(\hat{a}_{0}\right)\right]+\delta u^{0}\left(\hat{\sigma}_{1}\right) \geq(1-\delta) \bar{v}^{0}+\delta u^{0}(\sigma(0)) \Longleftrightarrow \hat{B}_{0} \geq \bar{v}^{0}-v^{0}(\hat{a})-\frac{\delta}{1-\delta}\left[u^{0}\left(\hat{\sigma}_{1}\right)-u^{0}(\sigma(0))\right]
$$

Next, look at principal j's deviation incentive at period 0 . However, we only have to check it only on the equilibrium path because, for the case where the agent has already deviated from $\hat{a}_{0}$, she plays the punishment strategy, paying nothing and following $\sigma(0)$, in which she has no deviation incentive by the assumption of the OPC. On the equilibrium path, if principal $j$ follows $\hat{\sigma}^{j}$ at period 0 , her utility can be expressed in a recursive way similar to the agent as

$$
(1-\delta)\left[v^{j}\left(\hat{a}_{0}\right)-\hat{b}_{0}^{j}\right]+\delta u^{j}\left(\hat{\sigma}_{1}\right)
$$

If she deviates to another payment $b^{\prime} \geq 0$, then she pays only $b^{\prime} \geq 0$ and since the players start the OPC on her from next period, her continuation payoff would be $u^{j}(\sigma(j))$. Then the utility is $(1-\delta)\left(v^{j}\left(\hat{a}_{0}\right)-b^{\prime}\right)+\delta u^{j}(\sigma(j))$. Notice that $(1-\delta)\left(v^{j}\left(\hat{a}_{0}\right)-b^{\prime}\right)+\delta u^{j}(\sigma(j)) \leq(1-\delta) v^{j}\left(\hat{a}_{0}\right)+\delta u^{j}(\sigma(j))$ for any $b^{\prime} \geq 0$. Thus principal $j$ does not deviate from $\hat{\sigma}$ at period 0 if and only if

$$
(1-\delta)\left[v^{j}\left(\hat{a}_{0}\right)-\hat{b}_{0}^{j}\right]+\delta u^{j}\left(\hat{\sigma}_{1}\right) \geq(1-\delta) v^{j}(\hat{a})+\delta u^{j}(\sigma(j)) \Longleftrightarrow \hat{b}_{0}^{j} \leq \frac{\delta}{1-\delta}\left[u_{1}^{j}\left(\hat{\sigma}_{1}\right)-u^{j}(\sigma(j))\right] .
$$

Then in order to check whether $\hat{\sigma}$ is SPE or not, the following conditions are necessary and sufficient. 
Lemma 2 Let $\hat{\sigma}$ be a strategy profile where $\left(\hat{a}_{0}, \hat{b}_{0}^{1}, \ldots, \hat{b}_{0}^{N}, \hat{\sigma}_{1}\right)$ is generated on the equilibrium path from $\hat{\sigma}$. Then it is a SPE if and only if

- equation (1) holds,

- equation (2) holds for $j \in \mathcal{N}$, and

- $\hat{\sigma}_{1} \in \hat{\Sigma}^{*}$.

For next sections, two kinds of the equilibria implemented by simple strategies will be characterized by abusing Lemma 2 . The first is on decision-stationary equilibria on which the agent chooses the same decision repeatedly. The second is the OPC. We first investigate the decisionstationary equilibria given the OPC $\{\sigma(i)\}_{i=0}^{N}$. While the characterization of decision-stationary equilibria is not be completed at this step since we do not know the OPC specifically, the result at this step helps us to characterize the OPC. Thus next we move to investigate the OPC and characterize it by using the result in the first step. Finally, after obtaining the OPC, the issue of decision-stationary equilibria is revisited to complete the characterization.

\section{Decision-Stationary Equilibria}

This section focus on the equilibria on which the agent chooses the same decision $\hat{a}$ every period. Formally, the definition is as follows.

Definition $1 \hat{\sigma}$ is a decision-stationary strategy profile of $\hat{a} \in \mathcal{A}$ if it generates the outcome path on which decision $\hat{a}$ is repeatedly chosen every period. $\hat{\Sigma}^{*}(\hat{a})$ is the set of decision-stationary SPE of $\hat{a}$ and $\hat{U}^{*}(\hat{a}):=\left\{\left(u^{0}(\hat{\sigma}), u^{1}(\hat{\sigma}), \ldots, u^{N}(\hat{\sigma})\right) \mid \hat{\sigma} \in \hat{\Sigma}^{*}(\hat{a})\right\}$, that is, the set of its payoff vectors.

At the present, we do not obtain the specific property of the OPC and then we just suppose that the OPC $\{\sigma(i)\}_{i=0}^{N}$ is already given for us. 
Consider a strategy profile $\hat{\sigma}$ which is decision-stationary of $\hat{a}$. By applying Lemma $2, \hat{\sigma}$ is a SPE if and only if

$$
\begin{aligned}
& \hat{\psi}(\hat{a}) \geq \bar{v}^{0}-v^{0}(\hat{a})-\frac{\delta}{1-\delta}\left[u^{0}\left(\hat{\sigma}_{1}\right)-u^{0}(\sigma(0))\right] \\
& \hat{\sigma}^{j}(\hat{a}) \leq \frac{\delta}{1-\delta}\left[u_{1}^{j}\left(\hat{\sigma}_{1}\right)-u^{j}(\sigma(j))\right], j \in \mathcal{N} \\
& \hat{\sigma}_{1} \in \hat{\Sigma}^{*}(\hat{a})
\end{aligned}
$$

where $\hat{\psi}(\hat{a}):=\sum_{k=1} \hat{\sigma}^{k}(\hat{a})$ and $\hat{\sigma}_{1}$ is the continuation strategy of $\hat{\sigma}$ on the equilibrium path. Note that $\hat{\sigma}_{1}$ must also be a decision-stationary SPE of $\hat{a}$.

Recall that we do not restrict some kinds of stationarity on payment schedule; the equilibrium payment at some period might be different from another period. Nevertheless we will now show that it is without loss of generality to restrict the payment schedule to being stationary for deriving the decision-stationary SPE.

The principal's strategy (or payment) is called stationary if the payment on the equilibrium path is determined only by the decision at the current period. The formal definition is as follows.

Definition 2 Let $\hat{\sigma}$ be a strategy profile which generates the equilibrium history $\left\{\hat{h}_{t}\right\}_{t=0}^{\infty}$. $\hat{\sigma}$ is paymentstationary of principal $j$ if $\hat{\sigma}^{j}$ can be described by

$$
\hat{\sigma}^{j}\left(\hat{h}_{t}, a\right)=\beta^{j}(a)
$$

for any $a \in \mathcal{A}$ and $\hat{h}_{t}$

$\hat{\sigma}$ is stationary if it is decision-stationary and payment-stationary.

So far, we have allowed the strategy on which the payment from the principals is history dependent. However, if we require payment-stationarity on the strategy profile, the principals' payment on the equilibrium path depend only on the agent's current decision. ${ }^{17)}$ The following

\footnotetext{
${ }^{17}$ We allow history-dependent payment off the equilibrium path even if the strategy profile is payment-stationary.
} 
proposition shows that any payoff of decision-stationary SPE can be replicated by stationary SPE.

Proposition 1 Suppose that $\hat{\sigma} \in \hat{\Sigma}^{*}(\hat{a})$. Then there exists a strategy profile which (i) is stationary, (ii) generates the same payoff vector, and (iii) is in $\hat{\Sigma}^{*}(\hat{a})$.

The previous literature of relational contracts (MacLeod and Malcomson (1989) and Levin (2003)) has already shown the "stationary contract representation". Proposition 1 shows that the stationary contract representation can be extended to common agency. ${ }^{18)}$ Proposition 1 allows us without loss of generality to restrict on stationary payments to characterize the set of the payoff vectors of decision-stationary equilibria, $\hat{U}^{*}(\hat{a})$.

When the strategy is stationary, the players would repeat the same stationary strategy $\hat{\sigma}$ for the continuation game every period, which makes the continuation payoff identical at any period. Then in the stationary strategy of decision $\hat{a}$, equation (3) and (4) are equivalent to

$$
\begin{array}{r}
\hat{\mathrm{B}}(\hat{a}) \geq \bar{v}^{0}-v^{0}(\hat{a})-\frac{\delta}{1-\delta}\left[u^{0}(\hat{\sigma})-u^{0}(\sigma(0))\right] \\
\hat{\beta}^{j}(\hat{a}) \leq \frac{\delta}{1-\delta}\left(u^{j}(\hat{\sigma})-u^{j}(\sigma(j))\right)
\end{array}
$$

where $\hat{\beta}^{j}(a)$ is the stationary payment of principal $j$ and $\hat{\mathrm{B}}(\cdot):=\sum_{j=1}^{N} \hat{\beta}^{j}(\cdot)$. Moreover, since the players would repeat the same stationary strategy every period, the one-shot net payoff is same among all periods and identical with the average payoff in the entire game, i.e. $v^{0}(\hat{a})+\hat{\mathrm{B}}(\hat{a})=u^{0}(\hat{\sigma})$ and $v^{j}(\hat{a})-\hat{\beta}^{j}(\hat{a})=u^{j}(\hat{\sigma})$ for $j \in \mathcal{N}$. Thus equation (5) and (6) are further reduced to

$$
\begin{aligned}
& u^{0}(\hat{\sigma}) \geq(1-\delta) \bar{v}^{0}+\delta u^{0}(\sigma(0)) \\
& \left.u^{j}(\hat{\sigma}) \geq(1-\delta) v^{j}(\hat{a})+\delta u^{j}(\sigma(j))\right) .
\end{aligned}
$$

\footnotetext{
18) It is worth noting that while the proof here is similar to Levin (2003), the limited liability might make his proof collapsed. The key idea of his proof is that an equilibrium on which the continuation play could depend on the public signal can be transformed to the equilibrium on which the agent can be incentivized only by the discretionary payoff. However under limited liability, this transformation might violate the limited liability constraint. Here we show that this limited liability does not matter when the information is perfect. We guess that as long as we focus on decision stationary equilibria, the limited liability is innocuous for stationary contract representation even if some kinds of asymmetric information (e.g. hidden action or hidden knowledge) are introduced.
} 
Finally, notice that due to that the players repeat the same stationary strategy every period, the continuation strategy is also a SPE if there are no incentives to deviate at period 0, which is equivalent to equation (7) and (8). With taking into consideration that the total net payoff among the players is always identical with $s(\hat{a})$ and $u^{j}(\hat{\sigma}) \leq v^{j}(\hat{a})$ for all $j \in \mathcal{N}$ because of the limited liability, the set of payoff vectors of decision-stationary equilibria is characterized as follows;

$$
\hat{U}^{*}(\hat{a})=\left\{\left(\begin{array}{c}
u^{0} \\
u^{1} \\
\vdots \\
u^{N}
\end{array}\right)^{\prime} \mid \begin{array}{l}
\sum_{j=0}^{N} u^{j}=s(\hat{a}), \\
u^{0} \geq(1-\delta) \bar{v}^{0}+\delta u^{0}(\sigma(0)), \\
v^{j}(\hat{a}) \geq u^{j} \geq(1-\delta) v^{j}(\hat{a})+\delta u^{j}(\sigma(j)),^{\forall} j \in \mathcal{N}
\end{array}\right\} .
$$

We cannot specify $\hat{U}^{*}(\hat{a})$ more because the OPC $\sigma(i)$ is not clear at the present. We will come back to this issue in section 6 after $\sigma(i)$ is made clear.

Instead, we will move to the other issue; the existence of (decision-)stationary equilibria of $\hat{a}$. The necessary condition of the existence is immediately derived by combining equation (5) and (6) to eliminate $\hat{\beta}^{j}(\hat{a})$, that is,

$$
\bar{v}^{0}-v^{0}(\hat{a})-\frac{\delta}{1-\delta}\left[u^{0}(\hat{\sigma})-u^{0}(\sigma(0))\right] \leq \sum_{j=1}^{N} \frac{\delta}{1-\delta}\left[u^{j}(\hat{\sigma})-u^{j}(\sigma(j)]\right.
$$

or, with $\sum_{j=0}^{N} u^{j}(\hat{\sigma})=s(\hat{a})$,

$$
\frac{\delta}{1-\delta}\left[s(\hat{a})-\sum_{j=0}^{N} u^{j}(\sigma(j))\right] \geq \bar{v}^{0}-v^{0}(\hat{a})
$$

Conversely, given equation (10), if we can construct $\hat{\beta}^{j}(\hat{a})$ which satisfies equation (7) and (8) (where $u^{j}(\hat{\sigma})=v^{j}(\hat{a})-\hat{\beta}^{j}(\hat{a})$ for $j \in \mathcal{N}$ and $\left.u^{0}(\hat{\sigma})=v^{0}(\hat{a})+\hat{\mathrm{B}}(\hat{a})\right)$, the stationary strategy of payment schedule $\left\{\hat{\beta}^{j}\right\}_{j=1}^{N}$ and decision $\hat{a}$ is a SPE, implying the existence of a decision-stationary SPE. Actually it can be shown and the following proposition is implied. 
Proposition 2 Given the OPC $\{\sigma(i)\}_{i=0^{\prime}}^{N}, \hat{\Sigma}^{*}(\hat{a}) \neq \emptyset$ if and only if equation (10) holds.

The sufficiency of equation (10) directly implies the following corollary about the existence of decision-stationary equilibria.

Corollary 1 1. If $\hat{\Sigma}^{*}\left(a^{\prime}\right) \neq \emptyset$ for some $a^{\prime} \in \mathcal{A}$, then $\hat{\Sigma}^{*}(\hat{a}) \neq \emptyset$ for all $\hat{a} \in \mathcal{A}$ such that

$$
\frac{\delta}{1-\delta} s(\hat{a})+v^{0}(\hat{a}) \geq \frac{\delta}{1-\delta} s\left(a^{\prime}\right)+v^{0}\left(a^{\prime}\right)
$$

2. If $\hat{\Sigma}^{*}\left(a^{\prime}\right)=\emptyset$ for some $a^{\prime} \in \mathcal{A}$, then $\hat{\Sigma}^{*}(\hat{a})=\emptyset$ for all $\hat{a} \in \mathcal{A}$ such that

$$
\frac{\delta}{1-\delta} s(\hat{a})+v^{0}(\hat{a})<\frac{\delta}{1-\delta} s\left(a^{\prime}\right)+v^{0}\left(a^{\prime}\right)
$$

3. $\hat{\Sigma}^{*}(\hat{a}) \neq \emptyset$ for all $\hat{a} \in \mathcal{A}$ if there exists $\bar{a}^{0} \in \bar{A}^{0}$ such that

$$
\frac{\delta}{1-\delta} s(\hat{a})+v^{0}(\hat{a}) \geq \frac{\delta}{1-\delta} s\left(\bar{a}^{0}\right)+\bar{v}^{0}
$$

The intuition is very simple. Rearranging equation (10) yields that

$$
\frac{\delta}{1-\delta} s(\hat{a})+v^{0}(\hat{a}) \geq \frac{\delta}{1-\delta} \sum_{j=0}^{N} u^{j}(\sigma(j))+\bar{v}^{0} .
$$

Notice that, given the OPC, the right hand side is fixed. Thus the greater the left hand side, the more likely to be possible to implement $\hat{a}$ it is. It is worth noting that the left hand side is the weighted sum of the agent benefit and the total surplus. Thus for ensuring the existence of the decision-stationary equilibrium, it is enough to assure that the decision generates large total surplus with large benefit for the agent.

Before concluding this section, we briefly discuss the socially efficient equilibrium. The efficient equilibrium is interesting to us because not only it could be a focal point, but also the strategy which generates the maximum total surplus helps us to investigate the OPC later. The following 
proposition states that it is without loss of generality that the efficient equilibrium is decisionstationary.

Proposition 3 If $\sigma$ is a SPE, there exists a strategy profile $\hat{\sigma}^{*}(\hat{a}) \in \hat{\Sigma}^{*}(\hat{a})$ such that $\sum_{i=0}^{N} u^{i}\left(\hat{\sigma}^{*}(\hat{a})\right) \geq$ $\sum_{i=0}^{N} u^{i}(\sigma)$.

The standard literature in relational contract provides us the the optimality of the stationary strategy. Proposition 3 says that the optimality of the stationary strategy can be extended to common agency ${ }^{19)}$ and we can restrict our attention to stationary strategy equilibria to look for the efficient equilibrium.

\section{The Optimal Penal Code}

In this section, we investigate the optimal penal code. Before the formal analysis, we will roughly present the conclusion. It is quite easy to get the OPC on the agent; it is just the Nash reversion. By contrast, the OPC on the principal is relatively complicated but still can be described in a simple way; in general, it would be the so-called two-phase scheme, first analyzed by Abreu (1986) in the model of tacit collusion in oligopoly.

\subsection{The Optimal Penal Code on the Agent}

The OPC on the agent first is quite simple. Recall that, beyond our model, the equilibrium payoff must be generally larger than or equal to the minimax value of the stage game. Then if there exists an equilibrium strategy by which the agent's payoff becomes his minimax value, it would be the OPC.

\footnotetext{
${ }^{19)}$ This is not trivial extension if the limited liability is imposed on the principals. Actually Fong and Li (2008) demonstrate bilateral relational contract model with limited liability under moral hazard in the employment context and show that the optimal relational contract for the principal is not a stationary contract. This is because, for the principal, the decision-stationary equilibrium is not optimal. Nevertheless, while we do not demonstrate formally here, we guess that the social optimal equilibria, which is more appealing in the context of common agency, can be achieved by a decision-stationary equilibrium even with limited liability under moral hazard.
} 
Now consider the stage game in our model. If the principals intend to minimize the agent payoff, they pay nothing. Given that, the agent would choose $\bar{a}^{0} \in \bar{A}^{0}$ so as to maximize his own payoff and gains $\bar{v}^{0}$, which is the minimax value for the agent. Actually, this is a subgame perfect equilibrium of the one-shot game. Thus the minimax payoff can be easily attained by the Nash reversion. Then we can simply get $u^{0}(\sigma(0))=\bar{v}^{0}$.

\subsection{The Optimal Penal Code on the Principals}

Let us look at the OPC on principal $k$. For a while, we still keep to suppose that the other's OPC have already been given for us.

Since $\sigma(k)$ is a SPE, it satisfies the conditions in Lemma 2. Let $\left(a_{0}(k),\left(b_{0}^{1}(k), \ldots, b_{0}^{N}(k)\right)\right)$ be the associated outcome path at period 0 generated by $\sigma(k)$ and $\sigma_{1}(k)$ be the continuation strategy from period 1 on the equilibrium path. Then by Lemma 2, $\sigma(k)$ is a SPE if and only if the following are satisfied;

$$
\begin{gathered}
(1-\delta)\left[v^{0}\left(a_{0}(k)\right)+B_{0}(k)\right]+\delta u^{0}\left(\sigma_{1}(k)\right) \geq \bar{v}^{0}, \\
b_{0}^{j}(k) \leq \frac{\delta}{1-\delta}\left[u^{j}\left(\sigma_{1}(k)\right)-u^{j}(\sigma(j))\right], j \in \mathcal{N},
\end{gathered}
$$

and $\sigma_{1}(k) \in \Sigma^{*}{ }^{20)}$

Since the OPC $\sigma(k)$ leads to the least SPE payoff for principal $j$, the $N+2$-tuple $\left(a_{0}(k),\left\{b_{0}^{j}(k)\right\}_{j=1}^{N}, \sigma_{1}(k)\right)$ can be characterized by the solution of the minimization problem where the objective is $u^{k}(\sigma(k)) \equiv$ $(1-\delta)\left[v^{k}\left(a_{0}(k)\right)-b_{0}^{k}(k)\right]+\delta u^{k}\left(\sigma_{1}(k)\right)$ subject to (12), (13) for $j \in \mathcal{N}$, and $\sigma_{1}(k) \in \Sigma^{*}$. Namely, it is the solution of the following program;

$$
\begin{aligned}
\min _{\left(a_{0}(k),\left\{b_{0}^{j}(k)\right\}_{j=1}^{N}, \sigma_{1}(k)\right)} & (1-\delta)\left[v^{k}\left(a_{0}(k)\right)-b_{0}^{k}(k)\right]+\delta u^{k}\left(\sigma_{1}(k)\right) \\
\text { subject to } & (12),(13), \sigma_{1}(k) \in \Sigma^{*}
\end{aligned}
$$

\footnotetext{
${ }^{20)}$ We take that $u^{0}(\sigma(0))=\bar{v}^{0}$ into account in equation (12).
} 
Now suppose that $b_{0}^{k}(k)<\delta\left(u^{k}\left(\sigma_{1}(k)\right)-u^{k}(\sigma(k))\right) /(1-\delta)$. Then, when we slightly increase $b_{0}^{k}(k)$ so as to keep to satisfy inequality (13) for $j=k$, the objective function (14) is decreasing without any violations on the constraints. Then it is not the solution, which implies that $b_{0}^{k}(k)=$ $\delta\left(u^{k}\left(\sigma_{1}(k)\right)-u^{k}(\sigma(k))\right) /(1-\delta)$. Substituting it into equation (14) yields the following result.

Lemma $3 u^{k}(\sigma(k))=v^{k}\left(a_{0}(k)\right)$ for all $k \in \mathcal{N}$.

Lemma 3 says that $u^{k}(\sigma(k))$ must be identical with the benefit at the first period on $\sigma(k)$. In other words, $u^{k}(\sigma(k))$ can be represented by the decision in $\mathcal{A}$ and we need to choose $a_{0}(k)$ which generates principal $k^{\prime}$ s benefit as small as possible, subject to the conditions for ensuring $\sigma(k)$ a SPE. Thus our problem can be simplified so as to minimize $v^{k}(a)$ where decision $a$ is associated with a pair of payments and a SPE strategy $\left(\left\{b_{0}^{j}(k)\right\}_{j \in \mathcal{N}}, \sigma_{1}(k)\right)$ such that $\left(a,\left\{b_{0}^{j}(k)\right\}_{j \in \mathcal{N}}, \sigma_{1}(k)\right)$ satisfies constraints (12), (13), and $\sigma_{1}(k) \in \Sigma^{*}$.

We have already seen that constraint (13) for $j=k$ is binding. Now look at constraint (13) for principal $j \neq k$. When $b_{0}^{j}(k)$ is not binding, increasing it slightly makes the degree of freedom in inequality (12) greater without any effects on the other constraints and the objective function. Then, without loss of generality, we can focus on the case where constraint (13) is binding at the upper bound for all $j \in \mathcal{N}$, i.e. $b_{0}^{j}(k)=\delta\left(u^{j}\left(\sigma_{1}(k)\right)-u^{i}(\sigma(k))\right) /(1-\delta) .{ }^{21)}$ Substituting them into constraint (12), we can reduce the constraints into $\sigma_{1}(k) \in \Sigma^{*}$ and

$$
(1-\delta) v^{0}\left(a_{0}(k)\right)+\delta\left[\sum_{j=1}^{N}\left(u^{j}\left(\sigma_{1}(k)\right)-u^{j}(\sigma(j))\right)\right]+\delta u^{0}\left(\sigma_{1}(k)\right) \geq \bar{v}^{0}
$$

Applying Lemma 3 for all $j \in \mathcal{N}$ and rearranging it yield that

$$
\frac{\delta}{1-\delta}\left[\sum_{i=0}^{N} u^{i}\left(\sigma_{1}(k)\right)-\left(\bar{v}^{0}+\sum_{j=1}^{N} v^{j}\left(a_{0}(j)\right)\right)\right] \geq \bar{v}^{0}-v^{0}\left(a_{0}(k)\right) .
$$

Furthermore, due to Proposition 3, if $a_{0}(k)$ satisfies this equation for some SPE $\sigma_{1}(k)$, it must satisfy

\footnotetext{
${ }^{21)}$ It is obvious that $b_{0}^{j}(k) \geq 0$ for all $i \in \mathcal{N}$.
} 
when $\sigma_{1}(k)$ is one of the decision-stationary SPE. Then without loss of generality we can restrict $\sigma_{1}(k)$ on decision-stationary SPE. Since the surplus is $s\left(a_{1}(k)\right)$ if $\sigma_{1}(k)$ is a decision stationary strategy of $a_{1}(k)$, this condition can be rewritten as

$$
\frac{\delta}{1-\delta}\left[s\left(a_{1}(k)\right)-\left(\bar{v}^{0}+\sum_{j=1}^{N} v^{j}\left(a_{0}(j)\right)\right] \geq \bar{v}^{0}-v^{0}\left(a_{0}(k)\right) .\right.
$$

Recall that thanks to Proposition 2, in order to check whether $\sigma_{1}(k)$ is a decision stationary SPE of $a_{1}(k)$ or not, equation (10) with replacing $\hat{a}$ by $a_{1}(k)$ is necessary and sufficient. Since $\sigma_{1}(k)$ must be a SPE, $a_{1}(k)$ must satisfy the following;

$$
\frac{\delta}{1-\delta}\left[s\left(a_{1}(k)\right)-\left(\bar{v}^{0}+\sum_{j=1}^{N} v^{j}\left(a_{0}(j)\right)\right)\right] \geq \bar{v}^{0}-v^{0}\left(a_{1}(k)\right) .
$$

To summarize, the problem for finding the OPC for principal $k$ is as follows;

\section{Problem $(k)$}

$$
\begin{array}{ll}
\min _{a_{0}(k), a_{1}(k)} & v^{k}\left(a_{0}(k)\right) \\
\text { subject to } & \frac{\delta}{1-\delta}\left[s\left(a_{1}(k)\right)-\left(\bar{v}^{0}+\sum_{j=1}^{N} v^{j}\left(a_{0}(j)\right)\right)\right] \geq \bar{v}^{0}-\min \left\{v^{0}\left(a_{0}(k)\right), v^{0}\left(a_{1}(k)\right)\right\} .
\end{array}
$$

Notice that the solution of Problem $(k)$ depends on $a_{0}(\ell)$ for $\ell \neq k$ which must solve Problem $(\ell)$. It means that the $2 n$-tuple $\left(a_{0}(1), a_{1}(1), a_{0}(2), a_{1}(2), \ldots, a_{0}(N), a_{1}(N)\right)$ must solve Problem $(k)$ for all $k \in \mathcal{N}$ simultaneously. However this $2 n$-tuple $\left(a_{0}(1), a_{1}(1), a_{0}(2), a_{1}(2), \ldots, a_{0}(N), a_{1}(N)\right)$ can be reduced to $n+1$-tuple $\left(a_{0}(1), a_{0}(2), \ldots, a_{0}(N), \hat{a}\right)$ thanks to the following lemma.

Lemma 4 Suppose that $2 n$-tuple $\left(a_{0}(1), a_{1}(1), a_{0}(2), a_{1}(2), \ldots, a_{0}(N), a_{1}(N)\right)$ solves Problem $(k)$ for all $k \in$ $\mathcal{N}$ simultaneously. Then without loss of generality there exists $\hat{a} \in \mathcal{A}$ such that $a_{1}(k)=\hat{a}$ for all $k \in \mathcal{N}$.

This lemma simplifies our problem into that the $n+1$-tuple $\left(a_{0}(1), a_{0}(2), \ldots, a_{0}(N), \hat{a}\right)$ minimizes 
$v^{k}\left(a_{0}(k)\right)$ for all $k \in \mathcal{N}$ simultaneously subject to only the following one constraint;

$$
\frac{\delta}{1-\delta}\left[s(\hat{a})-\left(\bar{v}^{0}+\sum_{j=1}^{N} v^{j}\left(a_{0}(j)\right)\right] \geq \bar{v}^{0}-\min \left\{v^{0}(\hat{a}), \min _{k \in \mathcal{N}} v^{0}\left(a_{0}(k)\right)\right\}\right.
$$

In words, the role of $\hat{a}$ is to relax the constraint so as to minimize $v^{k}\left(a_{0}(k)\right)$ for each $k \in \mathcal{N}$. To formalize this interpretation, denote $a(\mathcal{N}):=(a(1), \ldots, a(N)) \in \mathcal{A}^{N}$ and ${ }^{22)}$ define the sets as follows;

$$
\begin{aligned}
A_{1}^{P C}(\boldsymbol{a}(\mathcal{N})) & :=\left\{\hat{a} \in \mathcal{A} \mid \frac{\delta}{1-\delta}\left[s(\hat{a})-\left(\bar{v}^{0}+\sum_{j=1}^{N} v^{j}(a(j))\right)\right] \geq \bar{v}^{0}-\min \left\{v^{0}(\hat{a}), \min _{k \in \mathcal{N}} v^{0}(a(k))\right\}\right\}, \\
A_{0}^{P C} & :=\left\{\boldsymbol{a}(\mathcal{N}) \in \mathcal{A}^{N} \mid A_{1}^{P C}(\boldsymbol{a}(\mathcal{N})) \neq \emptyset\right\} .
\end{aligned}
$$

If $\boldsymbol{a}(\mathcal{N}) \in A_{0}^{P C}$, constraint $(15)$ can hold for $\boldsymbol{a}_{0}(\mathcal{N})=\boldsymbol{a}(\mathcal{N})$ and then $\{a(k)\}_{k=1}^{N}$ can be used as the candidate of the pair of the first period decision in each of the OPCs. Hence the pair of the first period decisions of the OPC payoff is chosen from $A_{0}^{P C}$ and determined in the following way.

Proposition $4\left(u^{1}(\sigma(1)), \ldots, u^{N}(\sigma(N))\right)=\left(v^{1}\left(a_{0}(1)\right), \ldots, v^{N}\left(a_{0}(N)\right)\right)$ where $a_{0}(\mathcal{N}) \in A_{0}^{P C}$ and for all $\boldsymbol{a}(\mathcal{N}) \in A_{0}^{P C}, v^{k}\left(a_{0}(k)\right) \leq v^{k}(a(k))$ for all $k \in \mathcal{N}$.

Notice that Proposition 4 says that the pair of the decisions $a_{0}(\mathcal{N})$ must (at least weakly) Pareto-dominate any pair of the decisions in $A_{0}^{P C}$ (in the negative sense). Then, in general, the existence of the pair satisfying Proposition 4 is not trivial because there are multiple points in the Pareto-frontier, which are never Pareto-dominated. ${ }^{23)}$ However it is assured in the following way.

Proposition 5 The pair $\boldsymbol{a}_{0}(\mathcal{N})$ satisfying the condition of Proposition 4 exists and if both $\boldsymbol{a}_{0}(\mathcal{N})$ and $\boldsymbol{a}_{0}(\mathcal{N})^{\prime}$ satisfy the condition, then $v^{k}\left(a_{0}(k)\right)=v^{k}\left(a_{0}(k)^{\prime}\right)$ for all $k \in \mathcal{N}$.

It is straightforward that if there exists $\left(\underline{a}^{1}, \ldots, \underline{a}^{N}\right) \in \prod_{j=1}^{N} \underline{A}^{j}$ such that $\left(\underline{a}^{1}, \ldots, \underline{a}^{N}\right) \in A_{0}^{P C}$, it is immediately the pair of the first decision in the OPCs. The condition for it can be simply expressed as follows.

\footnotetext{
${ }^{22)}$ Similarly, $\boldsymbol{a}_{0}(\mathcal{N}):=\left(a_{0}(1), \ldots, a_{0}(N)\right)$ and $\boldsymbol{a}(\mathcal{N})^{\prime}:=\left(a(1)^{\prime}, \ldots, a_{0}(N)^{\prime}\right)$ those appear later.

${ }^{23)}$ For example, if to decrease $v^{j}\left(a_{0}(j)\right)$ must induce to increase $v^{k}\left(a_{0}(k)\right)$, we must be faced with the trade-off between these two values which collapses Pareto dominance.
} 
Corollary 2 The optimal penal code satisfies $u^{j}(\sigma(j))=\underline{v}^{j}$ for all $j \in \mathcal{N}$ if and only if there exists a vector $\left(\hat{a}, \underline{a}_{1}, \ldots, \underline{a}_{N}\right) \in \mathcal{A} \times \prod_{j=1}^{N} \underline{A}^{j}$ such that

$$
\frac{\delta}{1-\delta}\left[s(\hat{a})-\left(\bar{v}^{0}+\sum_{j=1}^{N} \underline{v}^{j}\right)\right] \geq \bar{v}^{0}-\min \left\{v^{0}(\hat{a}), \min _{k \in \mathcal{N}} v^{0}\left(\underline{a}^{k}\right)\right\} .
$$

Notice that it is not straightforward that equation (16) always holds because the agent utility $v^{0}\left(\underline{a}^{j}\right)$ also does matter and if it is too costly, $\underline{a}^{j}$ is not feasible. For example, when $\delta=0$ as the extreme case, inequality (16) is simplified to $\min _{k} v^{0}\left(a_{0}(k)\right) \geq \bar{v}^{0}$, which implies that it is impossible to punish by any decision which generates a positive gain for the agent by deviation. However as $\delta$ is higher the more severe punishment is available and if $\delta$ is enough but feasibly large, equation (16) holds meaning that the first best OPC is possible.

Proposition 6 1. For all $k \in \mathcal{N}, v^{k}\left(a_{0}(k)\right)$ is nonincreasing in $\delta$.

2. There exists $\bar{\delta}^{\mathrm{OPC} *} \in[0,1)$ such that for $\delta \in\left[\bar{\delta}^{\mathrm{OPC} *}, 1\right), u^{k}\left(a_{0}(k)\right)=\underline{v}^{k}$ for all $k \in \mathcal{N}$.

So far we have discussed the punishment payoff. Let us discuss how the OPC is operated.

Recall that, on strategy $\sigma(j)$, the agent chooses decision $a_{0}(j)$ at the first period and strategy $\sigma_{1}(j)$ at the continuation periods, the latter of which is replaced with some decision-stationary strategy of, say, $\hat{a}$. Thus, in general, the punishment scheme consists of two phases, $a_{0}(j)$ and $\hat{a}$.

From equation (14) for $i=j$,

$$
u^{j}(\sigma(j))=(1-\delta)\left(v^{j}\left(a_{0}(j)\right)-b_{0}^{j}(j)\right)+\delta\left(v^{j}(\hat{a})-\hat{b}^{j}\right)
$$

where $\hat{b}^{j}$ is the stationary payment in the continuation strategy, which is without loss of generality due to Proposition 1. With Lemma 3, we obtain

$$
v^{j}\left(a_{0}(j)\right)=(1-\delta)\left(v^{j}\left(a_{0}(j)\right)-b_{0}^{j}(j)\right)+\delta\left(v^{j}(\hat{a})-\hat{b}^{j}\right)
$$


or, if $\delta>0,{ }^{24)}$ it is equivalently

$$
v^{j}\left(a_{0}(j)\right)=v^{j}(\hat{a})-\frac{(1-\delta)}{\delta} b_{0}^{j}(j)-\hat{b}^{j}
$$

Since the payment must be nonnegative, this equation implies that $v^{j}\left(a_{0}(j)\right) \leq v^{j}(\hat{a})$.

First, suppose that $v^{j}\left(a_{0}(j)\right)=v^{j}(\hat{a})$. It immediately implies that $b_{0}^{j}(j)=\hat{b}^{j}=0$. Then in the OPC, principal $j$ gains the same level of the benefit and pays nothing over time. This is expressed as Figure 1. Especially if $\hat{a}=a_{0}(j),{ }^{25)}$ the agent chooses the same decision and principal $j$ has to reconcile to be punished by the harsh decision over time. In this sense, it can be interpreted as that she is excluded from the other players and we can call this punishment strategy an "Exclusiontype" of punishment. ${ }^{26)}$

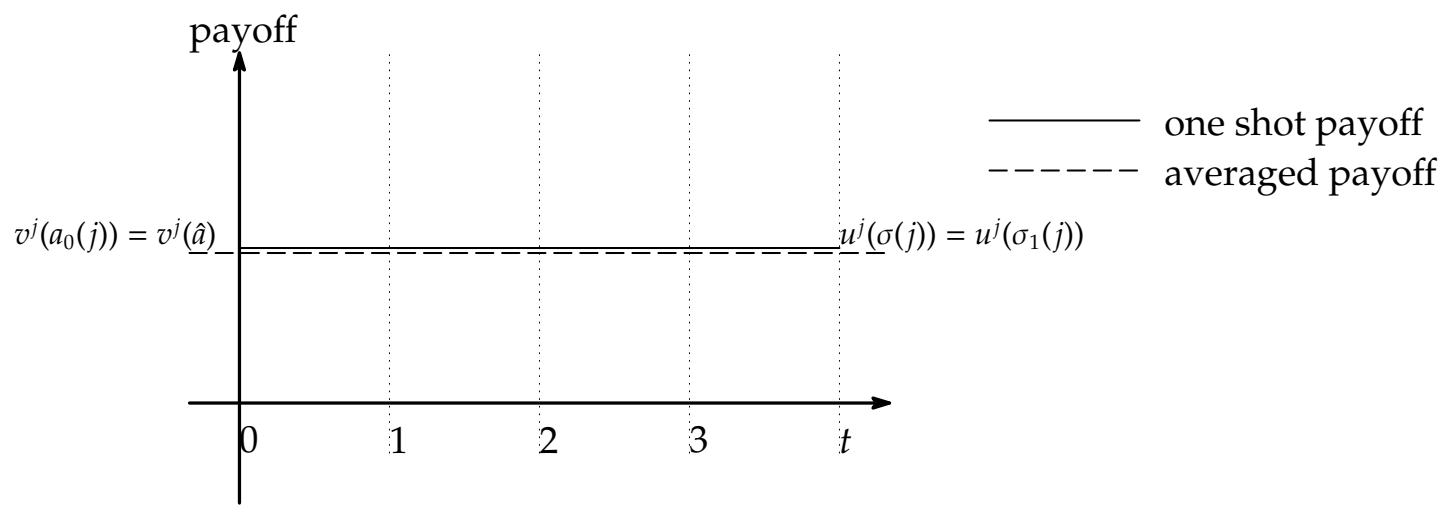

Figure 1: In Case of Exclusion-type $\sigma(j)$

Next suppose that $v^{j}\left(a_{0}(j)\right)<v^{j}(\hat{a})$. Note that

$$
\begin{aligned}
u^{j}\left(\sigma_{1}(j)\right)=v^{j}(\hat{a})-\hat{b}^{j}=v^{j}(\hat{a})-\left(v^{j}(\hat{a})-v^{j}\left(a_{0}(j)\right)-\frac{(1-\delta)}{\delta} b_{0}^{j}(j)\right) & =v^{j}\left(a_{0}(j)\right)+\frac{(1-\delta)}{\delta} b_{0}^{j}(j) \\
& \geq v^{j}\left(a_{0}(j)\right)=u^{j}(\sigma(j))
\end{aligned}
$$

\footnotetext{
${ }^{24)}$ If $\delta=0$, the OPC is just the Nash reversion, meaning that the agent repeatedly chooses decision in $\arg \max _{a \in A^{0}} v^{j}(a)$ and the principals pay nothing.

${ }^{25)}$ In general, $a_{0}(j)$ could be different from $\hat{a}$. However notice that because $\boldsymbol{a}_{0}(\mathcal{N}) \in A_{0}^{P C}$ and $v^{j}(\hat{a})=v^{j}\left(a_{0}(j)\right)$, $\left(a_{0}(0), \ldots, a_{0}(J-1), \hat{a}, a_{0}(J+1), \ldots, a_{0}(N)\right)$ is also in $A_{0}^{P C}$, which means that $\hat{a}$ can be also used as the first decision of $\sigma(j)$. Thus it is without loss of generality.

${ }^{26)}$ Even if $\hat{a} \neq a_{0}(j)$, the benefit for principal $j$ must kept be same as the possibly least level and she cannot do anything in the sense that she does not pay anything. Thus the notion of exclusion would be captured.
} 
and since $b_{0}^{j}(j) \geq 0$, we obtain $v^{j}\left(a_{0}(j)\right)-b_{0}^{j}(j) \leq v^{j}\left(a_{0}(j)\right)=u^{j}(\sigma(j))$. In words, the one-shot net payoff at the first period is less than the average punished utility, and the average utility in the continuation game must be more than the average punished utility in the entire game. This process can be seen as Figure 2. On the punishment path, principal $j$ is severely punished first and rewarded later. In this process, while she will incur the cost from the undesirable decision, she must additionally pay some amount of payment. It can be interpreted as the "sanction fine" or "consolation money" for deviation and, once she pays it, they go to the "normal" situation. In this sense, we call this punishment strategy a "Sanction-type" of punishment. This is qualitatively closely similar to the so-called "stick and carrot" strategy, where the first phase stands as the punishing "stick phase" and the remained phase as the rewarding "carrot phase", first analyzed by Abreu (1986) in the context of the tacit collusion in oligopolistic markets. ${ }^{27)}$

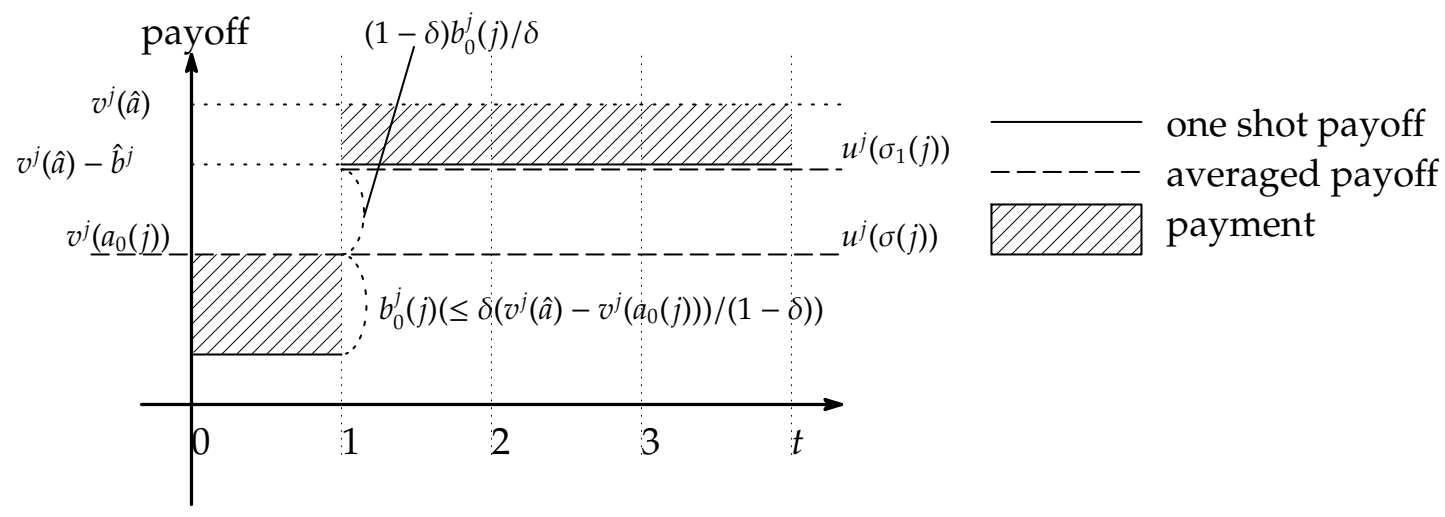

Figure 2: In Case of Sanction-type $\sigma(j)$

It is required to specify the payoff structure more to identify the first decision in the $\operatorname{OPC} a_{0}(j)$ more. We see the optimal punishment more by an example. Thereafter let $v^{j}\left(a_{0}(j)\right)$ be referred as the OPC payoff which is derived in this section.

\footnotetext{
${ }^{27)}$ Notice that if we pick $b_{0}^{j}(j)=0$, it is obtained that $\hat{b}^{j}=v^{j}(\hat{a})-v^{j}\left(a_{0}(j)\right)$ and then $u^{j}\left(\sigma_{1}(j)\right)=v^{j}(\hat{a})-\hat{b}^{j}=v^{j}\left(a_{0}(j)\right)=u^{j}(\sigma(j))$. In this case, principal $j$ is rewarded in the continuation periods in terms of the private benefit (i.e. rising up from $v^{j}\left(a_{0}(j)\right)$ to $\left.v^{j}(\hat{a})\right)$. However, because of the payment at the continuation stage $\hat{b}^{j}$, the one-shot net payoff keeps the same level over time as a result. Although the agent changes to the desirable decision for principal $j$ in the continuation period, she is still excluded in the sense of the net payoff level. This case might be considered as exclusion rather than sanction.
} 


\begin{tabular}{c|c|c|c} 
Decision & $v^{1}(a)$ & $v^{0}(a)$ & $v^{2}(a)$ \\
\hline \hline$a^{1}$ & $G^{1}$ & $-C$ & $-D$ \\
\hline$a^{0}$ & 0 & 0 & 0 \\
\hline$a^{2}$ & $-D$ & $-C$ & $G^{2}$
\end{tabular}

Table 1: Example

\subsection{Example}

We will demonstrate Sanction-type and Exclusion-type of punishment by the following example. Furthermore this example shows that depending on $\delta$, a Sanction-type can be equilibrium whereas an Exclusion-type cannot and vice-verse.

Suppose that $N=2$ and $\mathcal{A}=\left\{a^{0}, a^{1}, a^{2}\right\}$ and the private benefit for each player is given as Table 1 where $G^{1}, G^{2}, C$, and $D$ are all positive. We focus on the threshold of the discount factor above which $u^{j}(\sigma(j))=-D$ for $j=1,2$.

First, assume that that $G^{j}<C+D$ for $j=1,2$. Then $a^{0}$ is socially efficient and can be stationary decision equilibrium for any $\delta$. Thus from corollary $2, u^{j}(\sigma(j))=-D$ for $j=1,2$ if and only if

$$
\frac{\delta}{1-\delta}[0-(0-2 D)] \geq 0-\min \{0,-C\} \Longleftrightarrow \delta \geq \frac{C}{2 D+C}
$$

The question is whether the players can achieve this punishment by an Exclusion-type or not. If principal 2 is possible to be punished by an Exclusion-type of strategy, there must be a decisionstationary SPE of $a^{1}$ which is equivalent to, from equation (10),

$$
\frac{\delta}{1-\delta}\left[\left(G^{1}-D-C\right)-(0-2 D)\right] \geq 0-(-C) \Longleftrightarrow \delta \geq \frac{C}{G^{1}+D} .
$$

Notice that $C /\left(G^{1}+D\right)$ is greater than $C /(2 D+C)$. Then if $\delta \in\left[C /(2 D+C), C /\left(G^{1}+D\right)\right)$, the first best OPC can be achieved only through Sanction-types.

By contrast, we can obtain the opposite statement when the parametric assumption is changed. Now assume $G^{1}>C+D \geq G^{2}$. Then $a^{1}$ is the socially efficient decision. Given the punished 
payoff being $-D$ for both principals, $a^{1}$ can be implemented by a decision stationary SPE if and only if inequality (17) holds, i.e. $\delta \geq C /\left(G^{1}+D\right)$. Further, according to corollary 2 , this condition also provides the condition that the punishment payoff is $-D$. It implies that if $\delta \geq C /\left(G^{1}+D\right)$, principal 2 can be punished by an Exclusion-type of strategy such that $a^{1}$ is repeatedly chosen. The question is whether a Sanction-type is possible or not. For example, if the punished strategy is such that $a^{1}$ is chosen first and $a^{0}$ is repeatedly chosen after that, then inequality (15) for $\hat{a}=a^{0}$ must hold, that is,

$$
\frac{\delta}{1-\delta}[0-(0-2 D)] \geq 0-(-C) \Longleftrightarrow \delta \geq \frac{C}{2 D+C}
$$

Our hypothesis assures that $C /(2 D+C)$ is greater than $C /\left(G^{1}+D\right)$. Then if $\delta \in\left[C /\left(G^{1}+D\right), C /(2 D+C)\right)$, this Sanction-type of punishment can not attain the first best OPC. Similar analysis shows that with this discount factor, a Sanction-types of punishment such that $a_{2}$ is chosen from the second period can not attain the first best OPC neither. ${ }^{28)}$

The interesting implication from this example is that both Sanction-type and Exclusion-type could be a unique OPC, especially when the discount factor has an intermediate value. It is sharply contrasted with the context of optimal tacit collusion in oligopolistic markets. The characterization of the OPC here shares Abreu (1986)'s property in the sense that in general the OPC would be a two-phase scheme, the "punish" phase and the "reward" phase. In oligopolistic market, in order to punish the deviator credibly. at the punish phase, every firm must suffer from the punishment payoff by the predatory behaviour and then every firm must be rewarded later by the share of the monopolistic profit in the industry. It means that the two-phases are distinguishable. By contrast, in political contribution model, it is not necessary that all the players suffer in the punishment phase because there might be a decision which is quite terrible only for the punished principal and even such a decision is not much preferable for the agent, monetary transfer can adjust the

\footnotetext{
${ }^{28)}$ Precisely, this Sanction-type of punishment is impossible if and only if $\delta<C /\left(G^{2}+D\right)$ and it is easy to see that $C /\left(G^{2}+D\right)$ is greater than $C /(2 D+C)$.
} 
distribution of the total surplus so that the other principals can incentivize the agent to choose it. In the above example, this situation is clearly described when $G^{1}>C+D$ because decision $a^{1}$ is the worst for principal 2 but socially efficient and then principal 1 is willing to compensate the agent for choosing $a^{1}$ (if the players are somewhat patient). Because the agent and principal 1 has no benefit deviating from this situation, it would be repeated and then "exclusion" of principal 2 is going on.

\section{Characterization of Stationary Equilibrium Payoff}

This section goes back to the issue remained in section 4, the characterization of the set of decisionstationary equilibrium payoffs. We have already gotten the partial characterization of the decisionstationary equilibrium payoff in equation (9). With combining results about the OPC, one of our main results, the full characterization of the decision-stationary equilibrium payoff, is established as follows.

Proposition 7 Let $a_{0}(j)$ be the decision satisfying Proposition 4. Then

$$
\hat{U}^{*}(\hat{a})=\left\{\left(\begin{array}{c}
u^{0} \\
u^{1} \\
\vdots \\
u^{N}
\end{array}\right) \mid \begin{array}{l}
\sum_{j=0}^{N} u^{j}=s(\hat{a}), \\
u^{0} \geq \bar{v}^{0}, \\
v^{j}(\hat{a}) \geq u^{j} \geq(1-\delta) v^{j}(\hat{a})+\delta v^{j}\left(a_{0}(j)\right),{ }^{\forall} j \in \mathcal{N}
\end{array}\right\}
$$

We see two kinds of comparative statistics in $\delta$. First, as an extreme case, suppose that $\delta=0$. Then principal $j^{\prime}$ s net payoff must be exactly $v^{j}(\hat{a})$, which means that the principals pay nothing and the agent's net payoff must be $v^{0}(\hat{a})$. At the same time, the agent's net payoff must be greater than or equal to $\bar{v}^{0}$. Then it is the case (if and) only if $v^{0}(\hat{a}) \geq \bar{v}^{0}$, which implies that $\hat{a} \in \bar{A}^{0}$. Hence if $v^{0}(\hat{a})<\bar{v}^{0}, \hat{U}^{*}(\hat{a})$ is empty. This situation expresses the Nash reversion. 
The other extreme case is when $\delta$ is close to one. When $\delta$ is close to one, $v\left(a_{0}(j)\right)$ would be $\underline{v}^{j}$ by Proposition 6 and then $(1-\delta) v^{j}(\hat{a})+\delta v^{j}\left(a_{0}(j)\right)$ is close to $\underline{v}^{j}$, which is the minimum benefit (i.e. the minimax payoff) of principal $j$. Then if $\delta$ is close to 1 , given the sum of the utility fixed, any payoff vector larger than the minimax payoff can be supported on SPE. It is the similar argument to the folk theorem by Fudenberg and Maskin (1986). In Fudenberg and Maskin (1986), the sufficient condition for the folk theorem is about the dimensionality of the payoff matrix by which the players can punish and reward themselves in a sophisticated way. Here the transfer by the principals works for the sophisticated adjustment of the equilibrium payoff.

This characterization gives us another necessary condition for the existence of the stationary equilibrium. Suppose that for $\hat{a} \in \mathcal{N}$ and $\delta>0$, there exists principal $j$ such that $v^{j}\left(a_{0}(j)\right)>v^{j}(\hat{a})$. It immediately implies $(1-\delta) v^{j}(\hat{a})+\delta v^{j}\left(a_{0}(j)\right)>v^{j}\left(a_{0}(j)\right)$ and obviously there is no value $u^{j}$ such that $u^{j} \in\left[(1-\delta) v^{j}(\hat{a})+\delta v^{j}\left(a_{0}(j)\right), v^{j}\left(a_{0}(j)\right)\right]$. Then we cannot find any net payoff vectors of stationary equilibria of $\hat{a}$. This statement can also be proved for $\delta=0$.

Corollary 3 If there exists $j \in \mathcal{N}$ such that $v^{j}\left(a_{0}(j)\right)>v^{j}(\hat{a})$, then $\hat{\Sigma}^{*}(\hat{a})=\emptyset$.

\section{Validity of Menu Auction for Political Contribution}

We have established the payoff set of decision-stationary equilibria. Because the repetition of the same decision and payments realizes on decision-stationary equilibria, it can be compared with the one-shot common agency game by BW. We will compare the set of the equilibrium payoffs in our model with that of the static equilibrium payoffs.

\subsection{BW's Model}

For reader's convenience, we first describe the corresponding static model analyzed by BW. Hereafter we call it "Static Menu Auction (SMA)" and an equilibrium in it a SMA-equilibrium. By contrast, we will call our repeated game environment "Relational Political Contribution (RPC)" and a decision-stationary equilibrium of $a \in \mathcal{A}$ in it a RPC-equilibrium of $a$. 
SMA is the following one-shot game. First, each principal simultaneously and noncooperatively offers the contract which is the payment schedule contingent on the agent's decision and the agent accept it. ${ }^{29)}$ Second the agent makes a decision and the payment is enforced according to the binding contracts.

Let $\left(\hat{a},\left\{\left\{\hat{w}^{j}(a)\right\}_{a \in \mathcal{A}}\right\}_{j=1}^{N}\right)$ be the equilibrium decision and the payment contract contingent on the decisions in SMA. ${ }^{30)}$ BW derive the necessary and sufficient condition for SMA-equilibria.

Lemma 5 (BW, Lemma 2) $\left(\hat{a}, \hat{w}^{1}(\cdot), \ldots, \hat{w}^{N}(\cdot)\right)$ is a SMA-equilibrium if and only if the following conditions are satisfied;

1. $\hat{w}^{j}(a) \geq 0$ for all $a \in \mathcal{A}$ and $j=1, \ldots, N$

2. $\hat{a} \in \arg \max _{a}\left[v^{0}(a)+\hat{W}(a)\right]$

3. $v^{j}(\hat{a})+v^{0}(\hat{a})+\hat{W}^{-j}(\hat{a}) \geq v^{j}(a)+v^{0}(a)+\hat{W}^{-j}(a)$ for all $a \in \mathcal{A}$ and $j=1, \ldots, N$

4. for $j=1, \ldots, N$, there exists $a_{j} \in \arg \max _{a}\left[v^{0}(a)+\hat{W}(a)\right]$ such that $\hat{w}^{j}\left(a_{j}\right)=0$

where $\hat{W}(\cdot):=\sum_{i \in \mathcal{N}} w^{i}(\cdot)$ and $\hat{W}^{-j}(\cdot):=\sum_{i \in \mathcal{N} \backslash\{j\}} w^{i}(\cdot)$.

\subsection{Comparison between SMA and RPC}

Whereas the principals offer a binding contract which would be enforced ex post in SMA, the lobbyist's contract would be just implicit in realistic political situation. Hence when adopting SMA in political situation, we need a justification for the enforcement problem. If a SMA-equilibrium can be replicated by RPC-equilibria, the long term relationship between players can be a good micro-foundation for the political contribution in the SMA-equilibrium.. However, if it could not, we would realize the lack of the enforcement power of the agreement as a serious topic in political contribution model. Since the latter case is more interesting, we will investigate when the SMA-equilibrium can "not" be replicated.

\footnotetext{
${ }^{29)}$ Because we assume the nonnegative payment, the agent automatically accepts any offer.

${ }^{30)}$ Strictly, the description of the equilibrium should be the behaviour strategy of the agent rather than the decision chosen on the equilibrium path. As BW, however, we follow this description.
} 
Suppose that $\left(\hat{a}, \hat{w}^{1}(\cdot), \ldots, \hat{w}^{N}(\cdot)\right)$ is a SMA-equilibrium. Then the agent chooses $\hat{a}$ and principal $j \in \mathcal{N}$ pays $\hat{w}^{j}(\hat{a})$ to the agent on the equilibrium. Recall that, thanks to Proposition 1 , all the (decision-stationary) RPC-equilibria can be expressed by stationary strategies consisting of the decision and the payment on the path, say $\left(\hat{a},\left\{\hat{\beta}^{j}(\hat{a})\right\}_{j=1}^{N}\right)$. Thus if there exists a RPC-equilibrium such that $\hat{a}$ is chosen and $\hat{w}^{j}(\hat{a})$ is paid from principal $j$ to the agent, the net payoff vector of the SMA-equilibrium $\left(\hat{a}, \hat{w}^{1}(\cdot), \ldots, \hat{w}^{N}(\cdot)\right)$ can be replicated by the RPC-equilibrium.

Not surprisingly, when the players become less patient, a SMA-equilibrium is less likely to achieved by RPC equilibria. The following statement is the necessary and sufficient condition that a SMA-equilibrium cannot be replicated by RPC-equilibria.

Proposition 8 Let $\left(\hat{a}, \hat{w}^{1}(\cdot), \ldots, \hat{w}^{N}(\cdot)\right)$ be a SMA-equilibrium. Then given $\delta \in[0,1)$, the payoff vector $\left(\hat{y}^{0}, \hat{y}^{1}, \ldots, \hat{y}^{N}\right):=\left(v^{0}(\hat{a})+\hat{W}(\hat{a}), v^{1}(\hat{a})-\hat{w}^{1}(\hat{a}), \ldots, v^{N}(\hat{a})-\hat{w}^{N}(\hat{a})\right)$ cannot be supported by any RPCequilibria of $\hat{a}$ if and only if $\hat{w}^{j}(\hat{a})>\delta\left[v^{j}(\hat{a})-v^{j}\left(a_{0}(j)\right)\right]$ for some $j \in \mathcal{N}$.

The term $\delta\left[v^{j}(\hat{a})-v^{j}\left(a_{0}(j)\right)\right]$ is interpreted as the discounted benefit relative to deviation and the upper bound of the credible payment in RPC is determined by this value. If this value is not enough to compensate the payment on the SMA-equilibrium $\hat{w}^{j}(\hat{a})$, she cannot credibly pay this amount in RPC-equilibria. Note that this upper bound is weakly increasing in $\delta$ due to two effects; the direct effect through caring more about the future benefit (i.e. $\delta$ is increasing) ${ }^{31)}$ and the indirect effect through more severe punishment (i.e. $v^{j}\left(a_{0}(j)\right)$ is decreasing).

The next natural question is whether all SMA-equilibria can be supported by RPC equilibria if the discount factor is sufficiently high. Naively it seems to be true because, according to the folk theorem established in the repeated game theory, any individual rationally payoff vector can be achieved by subgame perfect equilibria if the discount factor is sufficiently high. However, somewhat surprisingly, it is not always the case. We first explain the reason by the term of the game theory. The strict statement of the folk theorem by Fudenberg and Maskin (1986) is that any "strict" individually rational payoff vector (i.e. the payoff vector each component of which is "strictly"

\footnotetext{
${ }^{31)}$ Recall that $v^{j}(\hat{a}) \geq v^{j}\left(a_{0}(j)\right)$ due to Corollary 3
} 
greater than its minimax value) can be achieved. In other words, the payoff vector some of the components of which contain the exact minimax value is not necessarily included in the argument of the folk theorem. However, in SMA, there might be an equilibrium where some principal gains her minimax payoff exactly and with some additional conditions, such payoff vector cannot be achieved in RPC for any $\delta \in[0,1)$. The formal statement of this notion is established as follows.

Proposition 9 Let $\left(\hat{a}, \hat{w}^{1}(\cdot), \ldots, \hat{w}^{N}(\cdot)\right)$ be a SMA-equilibrium. Then the payoff vector $\left(\hat{y}^{0}, \hat{y}^{1}, \ldots, \hat{y}^{N}\right):=$ $\left(v^{0}(\hat{a})+\hat{W}(\hat{a}), v^{1}(\hat{a})-\hat{w}^{1}(\hat{a}), \ldots, v^{N}(\hat{a})-\hat{w}^{N}(\hat{a})\right)$ cannot be supported by $R P C$-equilibria of $\hat{a}$ for any $\delta \in[0,1)$ if and only if there exists principal $j$ such that

$$
\hat{w}^{j}(\hat{a})=v^{j}(\hat{a})-\underline{v}^{j}>0
$$

It can be interpreted as a direct implication from Proposition 8. For principal $j$, the possible worst payoff is $\underline{v}^{j}$. Then the upper bound of the credible payment from principal $j$ is at most $v^{j}(\hat{a})-\underline{v}^{j}$ no matter how much $\delta$ is. Hence it is never individually rational to pay no less than $v^{j}(\hat{a})-\underline{v}^{j}$ to the agent for rewarding decision $\hat{a}$ chosen by the agent. Thus if a SMA-equilibrium requires principal $j$ to pay no less than $v^{j}(\hat{a})-\underline{v}^{j}$ on the equilibrium, it can never implemented in RPC-equilibria. By contrast, if the equilibrium payment in SMA is less than $v^{j}(\hat{a})-\underline{v}^{j}$ and the players are sufficiently patient, to punish by the minimax payoff $\underline{v}^{j}$ is credible and hence requiring principal $j$ to pay would be possible even in RPC.

So far we have referred any given $\left(\hat{a}, \hat{w}^{1}(\cdot), \ldots, \hat{w}^{N}(\cdot)\right)$ as a SMA-equilibrium and an important question whether there exists such a SMA-equilibrium which satisfies equation (18) remains. We will demonstrate that in some cases there exists such a SMA equilibrium. It implies that the commitment problem abstracted from SMA sometimes spoils the SMA equilibrium in political economy even the players are assumed to be sufficiently patient.

We illustrate it by truthful equilibria proposed by BW. 
Definition 3 The payment schedule $\hat{w}^{j}(\cdot)$ is truthful relative to $\hat{a}$ if $\hat{w}^{j}(\cdot)$ satisfies

$$
\hat{w}^{j}(a)=\max \left\{\hat{w}^{j}(\hat{a})+v^{j}(a)-v^{j}(\hat{a}), 0\right\}
$$

for all $a \in \mathcal{A}$. $\left(\hat{a}, \hat{w}^{1}(\cdot), \ldots, \hat{w}^{N}(\cdot)\right)$ is a truthful equilibrium in SMA (henceforth SMAT-equilibrium) if this is a SMA-equilibrium and $\hat{w}^{j}(\cdot)$ is truthful relative to $\hat{a}$ for all $j \in \mathcal{N}$.

BW show that SMAT-equilibria have some appealing properties; (i) it could be focal in the sense that there is always a truthful strategy which is the best response, (ii) SMAT-equilibria are (Pareto) efficient, and (iii) on SMAT-equilibria, the principals have no incentive to deviate jointly (i.e. coalition-proof). Because there typically exist many SMA-equilibria, many applied works adopt the SMAT-equilibrium as an equilibrium refinement. As we will see, however, this appealing equilibrium is sometimes vulnerable to the commitment problem.

Equation (18) consists of two conditions, $\hat{w}^{j}(\hat{a})=v^{j}(\hat{a})-\underline{v}^{j}$ and $v^{j}(\hat{a})>\underline{v}^{j}$. Recall that if $\hat{w}^{j}(\cdot)$ is a SMA-equilibrium contract, $\hat{w}(\hat{a})$ must be no more than $v^{j}(\hat{a})-\underline{v}^{j} \cdot{ }^{32)}$ Thus the first condition can be interpreted that principal $j$ pays the possibly most payment on the SMA-equilibrium. When we focus on SMAT-equilibria, such a large payment is obtained by the sufficient condition stated in the following lemma.

Lemma 6 Let $\left(\hat{a}, \hat{w}^{1}(\cdot), \ldots, \hat{w}^{N}(\cdot)\right)$ be a SMAT-equilibrium. Then $\hat{w}^{j}(\hat{a})=v^{j}(\hat{a})-\underline{v}^{j}$ if $\underline{A}^{j} \cap A^{*} \neq \emptyset$.

While the formal proof is left in the appendix, the sketch of it gives us the intuition. BW finds that a SMAT-equilibrium is closely related to the marginal contribution, that is roughly speaking how much the participation of the principal into a group raises the total value of the group. Specifically, BW show that the SMAT-equilibrium payoff of principal $j$ cannot be beyond the marginal contribution. Now if there exists a decision $\underline{a}^{j} \in \underline{A}^{j} \cap A^{*}$, choosing $\underline{a}^{j}$ maximizes the social surplus. Moreover since $\underline{a}^{j}$ minimizes principal $j$ 's benefit, the surplus in the coalition of

\footnotetext{
${ }^{32)}$ If $\hat{w}(\hat{a})>v^{j}(\hat{a})-\underline{v}^{j}$, the SMA-equilibrium payoff would be less than the minimax value $\underline{v}^{j}$, which cannot happen on any equilibria.
} 
all the players except for principal $j\left(\right.$ i.e. $\left.\sum_{i \neq j} v^{i}(a)\right)$ is also maximized. Then the principals except for $j$ wish to implement $\underline{a}^{j}$ and are willing to reward for $\underline{a}^{j}$ rather than the other decisions. Here principal $j$ has to pay much if she wants to avoid to be "isolated" by $\underline{a}^{j}$ chosen. In what follows, we call the set $\underline{A}^{j} \cap A^{*}$ or its element "completely isolating decision of $j$ ".

It is worth noting that the existence of the completely isolating decision is in general irrelevant to the equilibrium decision $\hat{a}$. More specifically, Lemma 6 does not necessary require that $\hat{a} \in \underline{A}^{j}$. As we will explain the detail later, the role of the completely isolating decision of $j$ is a credible threat on SMAT-equilibria to induce much payment from principal $j$.

Now suppose that there exist two principals, say $j$ and $k$, the completely isolating decisions of which exist, i.e. $\underline{A}^{j} \cap A^{*} \neq \emptyset$ and $\underline{A}^{k} \cap A^{*} \neq \emptyset$. If their preference is not congruent in the sense that they do not share the worst decisions for them, i.e. $\underline{A}^{j} \cap \underline{A}^{k}=\emptyset$, it is straightforward that $A^{*} \cap \underline{A}^{j} \cap \underline{A}^{k}=\emptyset$ which implies that for any $a^{*} \in A^{*}$, either $a^{*} \notin \underline{A^{j}}$ or $a^{*} \notin \underline{A}^{k}$ (or both). Since any SMAT-equilibrium decision must be socially efficient, i.e. $\hat{a} \in A^{*}$, we obtain either $v^{j}(\hat{a})>\underline{v}^{j}$ or $v^{k}(\hat{a})>\underline{v}^{k}$, implying that all possible SMAT-equilibria have principal $j$ or $k$ which satisfies equation (18).

Proposition 10 Suppose that there exist $j, k \in \mathcal{N}$ (where $j \neq k$ ) such that $\underline{A}^{j} \cap A^{*} \neq \emptyset, \underline{A}^{k} \cap A^{*} \neq \emptyset$ and $\underline{A}^{j} \cap \underline{A}^{k}=\emptyset$. Then for any $\delta \in[0,1)$ there are no RPC-equilibria which attain SMAT-equilibrium payoff vector.

To obtain the intuition, suppose that $N=2$. In SMA, it can be interpreted that reneging on the contract is exogenously punished by infinite amount of damage which makes it possible for the principals to commit any decision-contingent contract. Now suppose that there is a completely isolating decision of principal 2 and consider the joint benefit of the agent and principal 1. It is straightforward that they prefer the completely isolating decision of principal 2 the most and since there is no transaction cost such as asymmetric information and imperfect enforcement, they would readily implement the completely isolating decision of principal 2 unless principal 2 is there. This agreement works as a credible threat to fully exploit principal 2 by the agent. At the 
same time, the agent can make a same credible threat to principal 1 if there is a completely isolating decision of principal 1. Thus the agent can fully exploit both of the principals.

The full exploitation has either of the following form; i) the agent actually chooses the completely isolating decision or ii) the agent receives the most individually-rational amount of rent. If, for example, $\underline{A}^{1} \cap \underline{A}^{2}=\emptyset$ as the condition in Proposition 10, the latter type necessary happens on the SMAT-equilibrium because there is no completely isolating decision of both principal 1 and 2. However the full exploitation via transfer is impossible in RPC. Recall that, in RPC, reneging would be punished endogenously by the players and the punishment is delayed to start by one period implying that the punishment effect would be necessarily discounted. Then even if the punishment by the completely isolating decision is possible, the effect of the punishment is always discounted, which makes it impossible to exploit the most-individually-rational amount of rent in RPC. This interpretation exactly appears in the argument of the upper bound of the credible payment mentioned above. If there is an completely isolating decision of principal $j$, she is fully exploited by paying $v^{j}(\hat{a})-\underline{v}^{j}$ on the SMAT-equilibrium. However, the upper bound for principal $j$ on RPC-equilibria of $\hat{a}$ is at most $\delta\left[v^{j}(\hat{a})-\underline{v}^{j}\right]$ for any $\delta$. Then even the players can generate $\underline{v}^{j}$ as the punishment payoff of principal $j$, this effect would be discounted by $\delta$ due to the delay.

Under the condition in Proposition 10, principal $j$ and $k$ are in conflict via their completely isolating decisions. The idea that SMAT-equilibria are vulnerable due to conflict between the principals can also be confirmed by the following situation. Suppose that $s(a)=s^{*}$ for all $a \in \mathcal{A}$. In this case, the total surplus does not change at all and the decision determines only the distribution of the surplus between the players. Then this situation also captures the idea of conflict. The following proposition states that under this condition any non-trivial SMAT-equilibrium payoff in that the agent choice is indeed altered by the principals' payment schedule on the equilibrium cannot be supported by RPC-equilibria.

Proposition 11 Suppose that $s(a)=s^{*}$ for all $a \in \mathcal{A}$. Then any SMAT-equilibrium payoff on which the decision $\hat{a}$ is not in $\vec{A}^{0}$ cannot be supported by RPC-equilibria of $\hat{a}$ for any $\delta \in[0,1)$. 
Proposition 10 and 11 illustrate that the assumption of sufficient patience is sometimes not enough to justify for applying the SMA model in political economy, especially when conflict between players is severe.

\subsection{Example}

We revisit the example discussed in section 5, to demonstrate the results above and to provide some remarks. In the example, it is assumed that $N=2, \mathcal{A}=\left\{a^{0}, a^{1} \cdot a^{2}\right\}$, and the benefit is given by Table 1. Now further assume that $G^{1}=G^{2}=C+D$. Then it is not hard to derive a unique SMAT-equilibrium ${ }^{33)}$ as follows;

- $\left(\hat{w}_{0}^{j}, \hat{w}_{j}^{j}, \hat{w}_{k}^{j}\right)=(G-C, 2 G-C, 0)$ for $j, k=1,2$ and $j \neq k$

- the agent chooses $a^{0}$

where $G=G^{1}=G^{2}$ and $w_{k}^{j}$ be the payment from principal $j$ for the action $a^{k}$. The SMAT-equilibrium net payoff vector is given by $\left(\hat{y}^{0}, \hat{y}^{1}, \hat{y}^{2}\right)=(2(G-C),-G+C,-G+C)$.

This SMAT-equilibrium payoff cannot be achieved by any RPC-equilibria for any $\delta \in[0,1)$. Notice that it is obvious that $s\left(a^{0}\right)=s\left(a^{1}\right)=s\left(a^{2}\right)$ and $\hat{w}_{0}^{j}>0$, implying that the conditions in Proposition 11 hold. $^{34)}$ Put differently, recall that from Proposition 7 the lower bound of the RPC-equilibrium payoff of $a^{0}$ for principal $j$ is $(1-\delta) v^{j}\left(a^{0}\right)+\delta v^{j}\left(a_{0}(j)\right)$ which satisfies

$$
(1-\delta) v^{j}\left(a^{0}\right)+\delta v^{j}\left(a_{0}(j)\right)=(1-\delta) \dot{0}+\delta v^{j}\left(a_{0}(j)\right)=\delta v^{j}\left(a_{0}(j)\right) \geq-\delta D=-\delta(G-C)>-(G-C)
$$

for any $\delta \in[0,1)$. Then on all the RPC-equilibria of $a^{0}$, principal $j^{\prime}$ s net payoff must be strictly greater than the SMAT-equilibrium.

\footnotetext{
${ }^{33)}$ Bergemann and Välimäki (2003) propose the marginal contribution equilibrium and they show that if the game has a marginal contribution equilibrium, then the SMAT-equilibrium payoff is unique and coincides with that of the marginal contribution equilibrium. By applying this property, it is easy to see that the SMAT-equilibrium decision is uniquely given as $a^{0}$. See Bergemann and Välimäki (2003) for the detail of the marginal contribution equilibrium.

${ }^{34)}$ It is easy to confirm that the conditions in Proposition 9 and 10 also hold.
} 
We provide some remarks along with the example. First, although we see that every SMATpayoff can be approximately achieved by RPC with sufficiently patient players, it should not be interpreted that it is realistically valid. The minimum RPC-equilibrium net payoff of principal $j(=1,2)$ is $-\delta(G-C)$. It means that the SMA payoff can be approximately supported when $\delta$ is almost 1 . This statement is generally true because, as in the standard folk theorem, once the component of the net payoff vector can exceed the minimax value, it can be supported on RPCequilibria if $\delta$ is sufficiently high. However in some applications, to assume $\delta$ almost close to one seems to be unrealistic. ${ }^{35)}$ Additionally, if a SMA-equilibrium satisfies the conditions in Proposition 9, we see that for any $\delta \in[0,1)$, the set of RPC-equilibrium payoff of $\hat{a}, \hat{U}^{*}(\hat{a})$ is uniformly bounded from the SMA-equilibrium payoff vector. It means that for the commitment assumption plays a crucial role for the SMA-equilibrium.

The second remark is that although our argument seems to be weak in that equation (18) holds only in nongeneric cases, we should emphasize that the logic is robust. For instance, see again the above example with changing assumption that $0<G^{j}-C<D$ for $j=1$, 2. It is easily verified that the unique SMAT-equilibrium payoff is given by is given by $\left(\hat{y}^{0}, \hat{y}^{1}, \hat{y}^{2}\right)=\left(G^{1}+G^{2}-2 C,-G^{2}+C,-G^{1}+C\right)$ and it is replicated by RPC-equilibria of $a^{0}$ only if $\delta \geq \max \left\{\left(G^{1}-C\right) / D,\left(G^{2}-C\right) / D\right\} .{ }^{36)}$ Here $G^{j}-C$ is the joint surplus of the agent and principal $j$ and the larger it becomes, the more the agent can exploit principal $k(\neq j)$ in the SMAT-equilibrium. At the same time, however, if $G^{j}-C$ becomes larger, higher $\delta$ is required to achieve the SMAT outcome in the RPC environment. Needless to say, high $\delta$ is a stronger assumption than low $\delta$. In this sense, our claim would be valid. ${ }^{37)}$

Finally, whereas we focus on the relation of SMAT-equilibria and RPC-equilibria in Proposition 10 and 11, of course we can discuss the relation of SMA-equilibria (which is not necessarily truthful)

\footnotetext{
${ }^{35)}$ Many of the political positions has stipulates the finite length of the term of office. If the purpose of the lobbyists is to influence the decision made by the politician in office and the discount factor is interpreted as approximation of the length of the term of office, the discount factor would be regarded as lower.

36) Additionally, to assure the effective punishment, it is required that $\delta \geq C /(2 D+C)$. See section 5.3.

${ }^{37)}$ When $G^{j}-C$ is larger, the total surplus of $a^{j}$ is approaching that of $a^{0}$, meaning that the environment becomes more similar to one described in Proposition 11. It means that the logic behind Proposition 11 is also robust in the sense that higher $\delta$ is required to achieve the SMAT-equilibrium in the RPC environment if the total surplus are almost same between the decisions.
} 
and RPC-equilibria according to Proposition 9. For instance, consider again the above example and suppose that $G^{j}-C-D<0$ for $j=1,2$. Under this assumption, the following strategy profile is a SMA-equilibrium (which is not a SMAT-equilibrium);

- $\left(\hat{w}_{0}^{j}, \hat{w}_{j}^{j}, \hat{w}_{k}^{j}\right)=(D, 2 D+C, 0)$ for $j, k=1,2$ and $j \neq k$

- the agent chooses $a^{0}$.

It is easy to see that this SMA-equilibrium satisfies the condition in Proposition 9, implying that it cannot be supported on RPC-equilibria. Then there would be more environments on which SMA-equilibria cannot be supported on RPC-equilibria than those conditioned by Proposition 10 and 11.

\section{Conclusion}

This paper has formally analyzed the political contribution delivered via implicit agreements that is abstracted in most of the literature. Specifically, we have tried to find two issues. The first one is how to punish the deviating player credibly, by which the implicit agreement can be enforced. The second one is whether the outcome implied by explicit contracts is valid even by implicit agreements.

The punishment on a principal is more complicated than that in the existing relational contract framework because of the lack of the outside option. However it can be obtained by solving the minimization problem and the optimal punishment strategy is still simply described by the twophase strategy. It is further specified to either an Exclusion-type, on which the agent repeatedly chooses the decision harmful only for the deviating principal, or a Sanction-type, on which the harmful decision on the deviating principal is chosen only at the first period and then the beneficial decision is chosen.

We have derived the stationary equilibria and compared it with the equilibria in the static environment with explicit contracts. The main result is that the static equilibria sometimes cannot 
be attained by implicit contracts no matter how patient the players are. It suggests us that the commitment ability assumed in the static model is not necessarily justified only by the long-term relation between the players.

We conclude the paper by briefly mentioning remaining problems for future research. From a perspective of the literature of relational contracts, the model presented in this paper is just a first step toward common agency situation and still primitive. Introducing asymmetric information, heterogeneous discount factors, time-variant state variable, and multiple agents will provide an interesting extension. It will offer the concept of the equilibrium selection so that the multiplicity of the equilibria in our model can be mitigated. Additionally, when we depart from the political contribution by introducing the outside options, the difference between intrinsic common agency and delegated common agency seems to be an interesting issue. Specifically Martimort and Stole (2009) study this issue in static competition of nonlinear pricing and find that the property that the common agency game is either intrinsic or delegated has a different impacts on the market participation of consumers depending on whether the supplied goods are substitutes or complements. When price discrimination is operated by the relational contracts, we guess that the punishment issues have another effect on the equilibrium.

From the perspective of the dynamic politics by special interest groups, our model abstracts the usage of the political contribution. One of the primary purposes for the politician to get money would be the campaign advertisement to attract the naive voters. Thus introducing the election process in our framework would give us richer insights about interaction between campaign contribution and elections in dynamic situation. Specifically it includes how election process affects the campaign contribution via implicit agreements and how much such implicit agreements distort the election outcome from the median voter. Furthermore, the if the campaign promises are cheap talk so that it can be kept only by a reputation, ${ }^{38)}$ the politicians would face two kinds of reputation; for lobbyists to earn campaign contribution and for voters to get many votes. The

\footnotetext{
${ }^{38)}$ Aragonès et al. (2007) study this situation without lobbying activity.
} 
trade off of reputation building is an interesting issue.

\section{A Appendix: Proofs}

\section{A.1 Proof of Proposition 1}

For an arbitrary decision-stationary strategy $\hat{\sigma}$ of $\hat{a}$, let principal $j$ 's stationary strategy (payment schedule) be

$$
\hat{\beta}^{j}(\hat{a}):=\hat{\sigma}^{j}(\hat{a})+\frac{\delta}{1-\delta}\left[u^{j}(\hat{\sigma})-u^{j}\left(\hat{\sigma}_{1}\right)\right]
$$

and $\hat{\beta}^{j}(a)=0$ if $a \neq \hat{a}$, and consider the following stationary strategy profile;

- the agent always chooses $\hat{a}$ as long as no player has deviated,

- principal $j$ pays $\beta^{j}(\cdot)$ described above every period as long as no player has deviated,

and all players play the OPC once some player deviated. Since $u^{j}(\hat{\sigma})=(1-\delta)\left(v^{j}(\hat{a})-\hat{\sigma}^{j}(\hat{a})\right)+\delta u^{j}\left(\hat{\sigma}_{1}\right)$,

$$
\begin{aligned}
\hat{\beta}^{j}(\hat{a}) & =\hat{\sigma}^{j}(\hat{a})+\frac{\delta}{1-\delta}\left[u^{j}(\hat{\sigma})-u^{j}\left(\hat{\sigma}_{1}\right)\right] \\
& =\hat{\sigma}^{j}(\hat{a})+\frac{\delta}{1-\delta}\left[(1-\delta)\left(v^{j}(\hat{a})-\hat{\sigma}^{j}(\hat{a})\right)+\delta u^{j}\left(\hat{\sigma}_{1}\right)-u^{j}\left(\hat{\sigma}_{1}\right)\right] \\
& =\hat{\sigma}^{j}(\hat{a})+\delta\left[v^{j}(\hat{a})-\hat{\sigma}^{j}(\hat{a})-u^{j}\left(\hat{\sigma}_{1}\right)\right] \\
& \geq \hat{\sigma}^{j}(\hat{a})-\delta \hat{\sigma}^{j}(\hat{a}) \\
& =(1-\delta) \hat{\sigma}^{j}(\hat{a})
\end{aligned}
$$

where the inequality is due to decision stationarity of $\hat{\sigma}_{1}$ and the condition of non-negative payment, implying that $v^{j}(\hat{a}) \geq u^{j}\left(\hat{\sigma}_{1}\right)$. Note that since $\hat{\sigma}^{j}(\hat{a})$ must be non-negative, the constructed payment is also non-negative.

Since the present strategy profile is decision-stationary of $\hat{a}$ which is same as $\hat{\sigma}$, the total surplus does not change. Then if each of the principal's payoff is same as $\hat{\sigma}$, the agent's payoff is also same. 
Under this stationary contract, principal $j$ 's average payoff is

$$
\begin{aligned}
(1-\delta) \sum_{t=0}^{\infty} \delta^{t}\left[v^{j}(\hat{a})-\hat{\beta}^{j}(\hat{a})\right] & =v^{j}(\hat{a})-\hat{\beta}^{j}(\hat{a}) \\
& =v^{j}(\hat{a})-\hat{\sigma}^{j}(\hat{a})-\frac{\delta}{1-\delta}\left[u^{j}(\hat{\sigma})-u^{j}\left(\hat{\sigma}_{1}\right)\right] \\
& =\frac{1}{1-\delta}\left[(1-\delta)\left(v^{j}(\hat{a})-\hat{\sigma}^{j}(\hat{a})\right)+\delta u^{j}\left(\hat{\sigma}_{1}\right)-\delta u^{j}(\hat{\sigma})\right] \\
& =\frac{1}{1-\delta}\left[u^{j}(\hat{\sigma})-\delta u^{j}(\hat{\sigma})\right]=u^{j}(\hat{\sigma}) .
\end{aligned}
$$

Thus the payoff vector is same as $\hat{\sigma}$.

Finally, we will check that this stationary strategy is a SPE. Notice that the payoff vector is same as $\hat{\sigma}$ and if the players deviate at period 0 , the punished payoff is also same because they use the simple strategy. Then there is no incentive to deviate at period 0 if $\hat{\sigma}$ is SPE. Furthermore, since the chosen decision and payment schedule are completely identical at each period, the on-path continuation strategy profile is same among every periods. Then since there is no incentive to deviate at period 0 , there is either no incentive at the continuation periods. Thus the continuation strategy is also a SPE.

\section{A.2 Proof of Proposition 2}

We have already shown the necessity. Then it is enough to show the sufficiency. First of all, we will prove the following lemma.

Lemma 7 Given the OPC $\{\sigma(j)\}_{j=0^{\prime}}^{N}$ if $\hat{a} \in \mathcal{A}$ satisfies equation (10), $v^{j}(\hat{a}) \geq u^{j}(\sigma(j))$ for all $j \in \mathcal{N}$.

Proof (Lemma 7) Suppose that there exists principal $j$ such that $v^{j}(\hat{a})<u^{j}(\sigma(j))$. Specifically, we divide the set of the principals as follows.

$$
\underline{J}:=\left\{j \in \mathcal{N} \mid v^{j}(\hat{a})<u^{j}(\sigma(j))\right\}
$$


and $\bar{J}:=\mathcal{N} \backslash \underline{J}$. Notice that â satisfies equation (10), which is equivalent to

$$
\frac{\delta}{1-\delta}\left[\sum_{j \in \bar{J}}\left[v^{j}(\hat{a})-u^{j}(\sigma(j))\right]+\sum_{j \in \underline{J}}\left[v^{j}(\hat{a})-u^{j}(\sigma(j))\right]+v^{0}(\hat{a})-u^{0}(\sigma(0))\right] \geq \bar{v}^{0}-v^{0}(\hat{a}),
$$

or

$$
\delta\left[\sum_{j \in \bar{J}} v^{j}(\hat{a})-\sum_{j \in \bar{J}} u^{j}(\sigma(j))\right] \geq(1-\delta) \bar{v}^{0}+\delta u^{0}(\sigma(0))-v^{0}(\hat{a})-\delta \sum_{j \in \underline{J}}\left[v^{j}(\hat{a})-u^{j}(\sigma(j))\right]
$$

which, with the definition of $\mathrm{J}$, implies that

$$
\delta\left[\sum_{j \in \bar{J}} v^{j}(\hat{a})-\sum_{j \in \bar{J}} u^{j}(\sigma(j))\right] \geq(1-\delta) \bar{v}^{0}+\delta u^{0}(\sigma(0))-v^{0}(\hat{a}) .
$$

Now we will construct a stationary strategy which is a SPE. Here, however, we do not use the simple strategy. Instead we use the following modified simple strategy where the players ignore the deviation by principal $j \in$ J on the equilibrium path. Specifically, let

$$
\beta^{j}(\hat{a}):=\left\{\begin{array}{lll}
\delta\left[v^{j}(\hat{a})-u^{j}(\sigma(j))\right] & \text { for } & j \in \bar{J} \\
0 & \text { for } & j \in \underline{J}^{\prime}
\end{array}\right.
$$

$\beta^{j}(a)=0$ for all $a \neq \hat{a}$ and $j \in \mathcal{N}$, and $\mathrm{B}(\hat{a}):=\sum_{j \in \bar{J}} \beta^{j}(\hat{a})$. Notice that $\beta^{j} \geq 0$ for any $j \in \mathcal{N}$. The agent's strategy is

- to choose â at the first period and keep it if no players have deviated,

- to change to $\sigma^{0}(j)$ if principal $j \in \bar{J}$ deviated from paying $\beta^{j}(\hat{a})$, and

- to change to $\sigma^{0}(0)$ if the agent deviated from $\hat{a}$.

- (Even if principal $j^{\prime} \in J$ deviated from the on-equilibrium path, he keeps to choose $\hat{a}$ ).

Principal $j(\in J)$ 's strategy is

- to keep to pay $\beta^{j}(\hat{a})$ at each period if no players have deviated, 
- change to $\sigma^{j}\left(j^{\prime}\right)$, if principal $j^{\prime} \in \bar{J}$ deviated, and

- change to $\sigma^{0}(0)$ if the agent deviated from $\hat{a}$.

- (Even if principal $j^{\prime \prime} \in J$ deviated from the on-equilibrium path, she keeps to pay $\beta^{j}(\hat{a})$ ).

Notice that since we just assume that we know the OPC, the incentive on $\sigma(j)$ does not need to be checked.

Because the strategy is stationary, each of the players gets the same net payoff at each period $\left(v^{0}(\hat{a})+\mathrm{B}(\hat{a})\right.$ for the agent and $v^{j}(\hat{a})-\beta^{j}(\hat{a})$ for principal $\left.j\right)$ and their continuation payoff is also same at each period. Thus, in order to check that it is a SPE, we only have to check (i) the agent does not deviate at period 0 and (ii) each of the principals does not deviate at period 0 given $a_{0}=\hat{a}$.

The agent does not deviate at period 0 if and only if

$(1-\delta)\left(v^{0}(\hat{a})+\mathrm{B}(\hat{a})\right)+\delta\left(v^{0}(\hat{a})+\mathrm{B}(\hat{a})\right) \geq(1-\delta) \bar{v}^{0}+\delta u^{0}(\sigma(0)) \Longleftrightarrow \mathrm{B}(\hat{a}) \geq(1-\delta) \bar{v}^{0}+\delta u^{0}(\sigma(0))-v^{0}(\hat{a})$

which is equivalent to equation (19). Then the agent does not deviate at period 0.

For principal $j \in \bar{J}$, she does not deviate at period 0 if and only if

$(1-\delta)\left(v^{j}(\hat{a})-\beta^{j}(\hat{a})\right)+\delta\left(v^{j}(\hat{a})-\beta^{j}(\hat{a})\right) \geq(1-\delta)\left(v^{j}(\hat{a})-0\right)+\delta u^{j}(\sigma(j)) \Longleftrightarrow \beta^{j}(\hat{a}) \leq \delta\left[v^{j}(\hat{a})-u^{j}(\sigma(j))\right]$

which holds by the construction of $\beta^{j}(\hat{a})$.

Finally for principal $j \in J$, when she follows $\beta^{j}(\hat{a})=0$, her payoff is $v^{j}(\hat{a})$. Moreover even if she deviates to $b^{j \prime}>0$, her continuation payoff does not change from $v^{j}(\hat{a})$ because the punishment is not starting. Then her payoff is

$$
(1-\delta) v^{j}(\hat{a})-b^{j \prime}+\delta v^{j}(\hat{a})=v^{j}(\hat{a})-b^{j \prime}
$$

which is strictly less than the payoff without deviating for any $b^{j \prime}>0$. Hence she prefers to follow this strategy. 


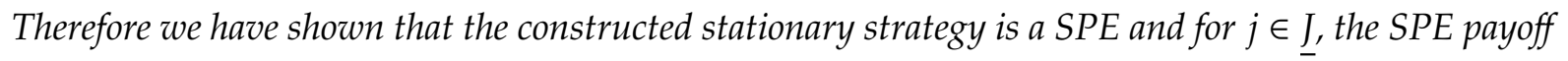
is $v^{j}(\hat{a})$. which contradicts the hypothesis $v^{j}(\hat{a})<u^{j}(\sigma(j))$ we supposed first.

Suppose that equation (10) holds. Now consider the following stationary strategy (with denoting $\hat{\sigma})$;

- the agent always chooses $\hat{a}$ as long as no players have deviated,

- principal $j$ pays $\beta^{j}(\cdot)$ (described below) every period as long as no players have deviated,

and all players play the OPC once some player deviated, where, for $j \in \mathcal{N}$,

$$
\beta^{j}(\hat{a}):=\delta\left[v^{j}(\hat{a})-u^{j}(\sigma(j))\right]
$$

and $\beta^{j}(a)=0$ for all $a \neq \hat{a}$. Notice that due to Lemma 7 , it is nonnegative.

Because the strategy is stationary, each of the players gets the same net payoff at each period and it is identical with the average payoff. Then $u^{0}(\hat{\sigma})=v^{0}(\hat{a})+\mathrm{B}(\hat{a})$ where $\mathrm{B}(\cdot)=\sum_{k=1} \beta^{k}(\cdot)$ and $u^{j}(\hat{\sigma})=v^{j}(\hat{a})-\beta^{j}(\hat{a})$ for $j \in \mathcal{N}$. Thus

$$
\begin{aligned}
u^{0}(\hat{\sigma})-(1-\delta) \bar{v}^{0}-\delta u^{0}(\sigma(0)) & =v^{0}(\hat{a})+\mathrm{B}(\hat{a})-(1-\delta) \bar{v}^{0}-\delta u^{0}(\sigma(0)) \\
& =v^{0}(\hat{a})+\sum_{j=1}^{N} \delta\left[v^{j}(\hat{a})-u^{j}(\sigma(j))\right]-(1-\delta) \bar{v}^{0}-\delta u^{0}(\sigma(0)) \\
& =(1-\delta) v^{0}(\hat{a})+\delta\left[s(\hat{a})-\sum_{j=0}^{N} u^{j}(\sigma(j))\right]-(1-\delta) \bar{v}^{0} \\
& =(1-\delta)\left[\frac{\delta}{1-\delta}\left(s(\hat{a})-\sum_{j=0}^{N} u^{j}(\sigma(j))\right) v^{0}(\hat{a})-\bar{v}^{0}\right] \geq 0
\end{aligned}
$$

where the last inequality is due to equation (10). It implies that equation (7) holds. For $j \in \mathcal{N}$,

$$
\begin{aligned}
\left.u^{j}(\hat{\sigma})-(1-\delta) v^{j}(\hat{a})-\delta u^{j}(\sigma(j))\right) & \left.=v^{j}(\hat{a})-\beta^{j}(\hat{a})-(1-\delta) v^{j}(\hat{a})-\delta u^{j}(\sigma(j))\right) \\
& \left.=v^{j}(\hat{a})-\delta\left[v^{j}(\hat{a})-u^{j}(\sigma(j))\right]-(1-\delta) v^{j}(\hat{a})-\delta u^{j}(\sigma(j))\right)=0
\end{aligned}
$$


and $v^{j}(\hat{a})-u^{j}(\hat{\sigma})=\beta^{j}(\hat{a}) \geq 0$ those imply that equation (8) holds.

Therefore, since the stationary strategy satisfy equation (7) and equation (8), it is a SPE, implying the existence.

\section{A.3 Proof of Corollary 1}

1 and 2. Since decision $a^{\prime}$ satisfies (resp. does not satisfy) equation (10), decision $\hat{a}$ satisfies (resp. does not satisfy) equation (10) and by Proposition 2, $\hat{a}$ can (resp. cannot) be implemented.

3. Notice that decision $\bar{a}^{0} \in \bar{A}^{0}$ can be always implemented by Nash reversion, which implies that equation (10) holds for $\hat{a}=\bar{a}^{0}$. Then the previous argument can be applied.

\section{A.4 Proof of Proposition 3}

Suppose that $\sigma$ is a SPE and the associated equilibrium decision and payment path are given by $\left.\left(\left(a_{0}, a_{1}, \ldots\right),\left(b_{0}^{1}, \ldots, b_{0}^{N}\right),\left(b_{1}^{1}, \ldots, b_{1}^{N}\right), \ldots\right)\right)$. Now define a sequence $\left\{\tilde{a}_{t}\right\}_{t=0}^{\infty}$ as follows; $\tilde{a}_{0}:=a_{0}$ and

$$
\tilde{a}_{t}=\left\{\begin{array}{lll}
a_{t} & \text { if } & s\left(a_{t}\right) \geq s\left(\tilde{a}_{t-1}\right) \\
& & \text { otherwise. }
\end{array}\right.
$$

Note that by construction $s\left(\tilde{a}_{t}\right) \geq s\left(\tilde{a}_{t-1}\right)$ for all $t \geq 0$. Since $\mathcal{A}$ is compact, by Bolzano-Weierstrass theorem, we can choose a converging subsequence $\left\{\tilde{a}_{t}^{\prime}\right\}_{t=0}^{\infty}$ of $\left\{\tilde{a}_{t}\right\}_{t=0}^{\infty}$. Let $\tilde{a}^{\prime}:=\lim _{t \rightarrow \infty} \tilde{a}_{t}^{\prime}$. Now we will show that;

Claim $1 s\left(\tilde{a}^{\prime}\right) \geq \sum_{j=0}^{N} u^{j}\left(\sigma_{t}\right)$ for all $t \geq 0$ where $\sigma_{t}$ is the on-path continuation strategy of $\sigma$ at period $t$.

Claim $2 \quad \hat{\Sigma}^{*}\left(\tilde{a}^{\prime}\right) \neq \emptyset$.

These claims imply that there exists a decision-stationary equilibrium of $\tilde{a}^{\prime}$ which generates the total surplus at least $\sum_{j=0}^{N} u^{j}(\sigma)$.

Proof of Claim 1 Notice the following observation; 
1. by construction of $\tilde{a}_{t}, s\left(\tilde{a}_{t}\right) \geq s\left(a_{t}\right)$ for all $t \geq 0$.

2. since $\left\{\tilde{a}_{t}^{\prime}\right\}_{t=0}^{\infty}$ is a subsequence of $\left\{\tilde{a}_{t}\right\}_{t=0}^{\infty}, s\left(\tilde{a}_{t}^{\prime}\right) \geq s\left(\tilde{a}_{t}\right)$ and $s\left(\tilde{a}_{t}^{\prime}\right) \geq s\left(\tilde{a}_{t-1}^{\prime}\right)$ for all $t \geq 0$.

These imply that $s\left(\tilde{a}_{t}^{\prime}\right) \geq s\left(a_{t}\right)$ for all $t \geq 0$. Furthermore because $s(\cdot)$ is continuous and bounded and $\left\{s\left(\tilde{a}_{t}^{\prime}\right)\right\}_{t=0}^{\infty}$ is a bounded and nondecreasing sequence, we obtain that for any $t \geq 0$

$$
s\left(\tilde{a}_{t}^{\prime}\right) \leq \sup _{k \geq 0} s\left(\tilde{a}_{k}^{\prime}\right)=\lim _{k \rightarrow \infty} s\left(\tilde{a}_{k}^{\prime}\right)=s\left(\lim _{k \rightarrow \infty} \tilde{a}_{k}^{\prime}\right)=s\left(\tilde{a}^{\prime}\right),
$$

implying that $s\left(a_{t}\right) \leq s\left(\tilde{a}^{\prime}\right)$ for any $t \geq 0$. It yields that

$$
\begin{aligned}
\sum_{j=0}^{N} u^{j}\left(\sigma_{t}\right) & =\sum_{j=0}^{N}\left[(1-\delta) \sum_{k=t}^{\infty} \delta^{k-t} v^{j}\left(a_{k}\right)\right] \\
& =(1-\delta) \sum_{k=t}^{\infty} \sum_{j=0}^{N} \delta^{k-t} v^{j}\left(a_{k}\right) \\
& =(1-\delta) \sum_{k=t}^{\infty} \delta^{k-t} s\left(a_{k}\right) \\
& \leq(1-\delta) \sum_{k=t}^{\infty} \delta^{k-t} s\left(\tilde{a}^{\prime}\right)=s\left(\tilde{a}^{\prime}\right) .
\end{aligned}
$$

Proof of Claim 2 Since $\sigma$ is a SPE, neither the agent nor the principals deviate at any period $t$ which is described as following;

$$
(1-\delta)\left(v^{0}\left(a_{t}\right)+B_{t}\right)+\delta u^{0}\left(\sigma_{t+1}\right) \geq(1-\delta)\left(\max _{a_{t} \in \mathcal{A}} v^{0}\left(a_{t}\right)+0+\delta u^{0}(\sigma(0))\right)=(1-\delta) \bar{v}^{0}+\delta u^{0}(\sigma(0))
$$

for the agent and

$$
(1-\delta)\left(v^{j}\left(a_{t}\right)-b_{t}^{j}\right)+\delta u^{j}\left(\sigma_{t+1}\right) \geq(1-\delta)\left(v^{j}\left(a_{t}\right)-0\right)+\delta u^{j}(\sigma(j))
$$

for $j \in \mathcal{N}$. Combining them to eliminate $b_{t}^{j}$ implies that

$$
\frac{\delta}{1-\delta}\left[\sum_{j=0}^{N} u^{j}\left(\sigma_{t+1}\right)-\sum_{j=0}^{N} u^{j}(\sigma(j))\right] \geq \bar{v}^{0}-v^{0}\left(a_{t}\right)
$$


for all $t$. Because $\sum_{j=0}^{N} u^{j}\left(\sigma_{t+1}\right) \leq s\left(\tilde{a}^{\prime}\right)$ by Claim 1 and $\tilde{a}_{t}^{\prime}=a_{s}$ for some $s \geq 0$, we obtain that

$$
\frac{\delta}{1-\delta}\left[s\left(\tilde{a}^{\prime}\right)-\sum_{j=0}^{N} u^{j}(\sigma(j))\right] \geq \bar{v}^{0}-v^{0}\left(\tilde{a}_{t}^{\prime}\right)
$$

for all $t \geq 0$. Since $\left\{\tilde{a}_{t}^{\prime}\right\}_{t=0}^{\infty}$ is a converging sequence to $\tilde{a}^{\prime}$ and $v^{0}(\cdot)$ is continuous, the both sides are converging. Then the inequality still holds at the limit, which means that

$$
\frac{\delta}{1-\delta}\left[s\left(\tilde{a}^{\prime}\right)-\sum_{j=0}^{N} u^{j}(\sigma(j))\right] \geq \bar{v}^{0}-\lim _{t \rightarrow \infty} v^{0}\left(\tilde{a}_{t}^{\prime}\right)=\bar{v}^{0}-v^{0}\left(\tilde{a}^{\prime}\right)
$$

and then by Proposition 2, we obtain Claim 2.

\section{A.5 Proof of Lemma 4}

For $2 n$-tuple $\left(a_{0}(1), a_{1}(1), a_{0}(2), a_{1}(2), \ldots, a_{0}(N), a_{1}(N)\right)$ which solves Problem $(k)$ for all $k \in \mathcal{N}$ simultaneously, choose $\ell \in \arg \max _{j \in \mathcal{N}} s\left(a_{1}(j)\right)$. Notice that for all $k \in \mathcal{N}, a_{1}(k)$ does not appear in the objective function and the role of it is only to satisfy the constraint. Then it is enough to check that the same $2 n$-tuple but $a_{1}(j)=a_{1}(\ell)$ for any $j \neq \ell$ satisfies the constraints of Problem $(k)$ for all $k \in \mathcal{N}$.

Recall that $a_{1}(j)$ appears only in the constraint of Problem $(j)$. Then it is enough to show that for all $j \in \mathcal{N} \backslash\{\ell\}$,

$$
\frac{\delta}{1-\delta}\left[s\left(a_{1}(\ell)\right)-\left(\bar{v}^{0}+\sum_{i=1}^{N} v^{i}\left(a_{0}(i)\right)\right)\right] \geq \bar{v}^{0}-\min \left\{v^{0}\left(a_{0}(j)\right), v^{0}\left(a_{1}(\ell)\right)\right\}
$$

Since $a_{1}(\ell)$ satisfies the constraint of Problem $(\ell)$,

$$
\begin{aligned}
\frac{\delta}{1-\delta}\left[s\left(a_{1}(\ell)\right)-\left(\bar{v}^{0}+\sum_{i=1}^{N} v^{i}\left(a_{0}(i)\right)\right)\right] & \geq \bar{v}^{0}-\min \left\{v^{0}\left(a_{0}(\ell)\right), v^{0}\left(a_{1}(\ell)\right)\right\} \\
& \geq \bar{v}^{0}-v^{0}\left(a_{1}(\ell)\right)
\end{aligned}
$$


and since $s\left(a_{1}(\ell)\right) \geq s\left(a_{1}(j)\right)$ and $a_{0}(j)$ and $a_{1}(j)$ satisfy the constraint of Problem $(j)$,

$$
\begin{aligned}
\frac{\delta}{1-\delta}\left[s\left(a_{1}(\ell)\right)-\left(\bar{v}^{0}+\sum_{i=1}^{N} v^{i}\left(a_{0}(i)\right)\right)\right] & \geq \frac{\delta}{1-\delta}\left[s\left(a_{1}(j)\right)-\left(\bar{v}^{0}+\sum_{i=1}^{N} v^{i}\left(a_{0}(i)\right)\right)\right] \\
& \geq \bar{v}^{0}-\min \left\{v^{0}\left(a_{0}(j)\right), v^{0}\left(a_{1}(j)\right)\right\} \\
& \geq \bar{v}^{0}-v^{0}\left(a_{0}(j)\right) .
\end{aligned}
$$

The two inequalities imply the desired result.

\section{A.6 Proof of Proposition 5}

For any $\boldsymbol{a}(\mathcal{N}), \boldsymbol{a}(\mathcal{N})^{\prime} \in A_{0}^{P C}$, denote $\boldsymbol{a}(\mathcal{N})^{\prime \prime}:=\left(a(1)^{\prime \prime}, \ldots, a(N)^{\prime \prime}\right)$ where $a(j)^{\prime \prime} \in \arg \min _{a \in\left\{a(j), a(j)^{\prime}\right\}} v^{j}(a)$ for all $j \in \mathcal{N}$.

The following lemma is useful to show the result.

Lemma $8 a(\mathcal{N})^{\prime \prime} \in A_{0}^{P C}$.

Proof (Lemma 8) Since $\boldsymbol{a}(\mathcal{N}) \in A_{0}^{P C}$, for any $\hat{a} \in A_{1}^{P C}(\boldsymbol{a}(\mathcal{N}))$, the followings must hold;

$$
\begin{aligned}
& \frac{\delta}{1-\delta}\left[s(\hat{a})-\left(\bar{v}^{0}+\sum_{i=1}^{N} v^{i}(a(i))\right)\right] \geq \bar{v}^{0}-\min _{k \in \mathcal{N}} v^{0}(a(k)) \\
& \frac{\delta}{1-\delta}\left[s(\hat{a})-\left(\bar{v}^{0}+\sum_{i=1}^{N} v^{i}(a(i))\right)\right] \geq \bar{v}^{0}-v^{0}(\hat{a}) .
\end{aligned}
$$

Because of the definition of $\boldsymbol{a}(\mathcal{N})^{\prime \prime}$, we obtain

$$
\sum_{i=1}^{N} v^{i}\left(a(i)^{\prime \prime}\right) \leq \sum_{i=1}^{N} v^{i}(a(i))
$$

which implies that, with equation (21) and (22),

$$
\begin{aligned}
& \frac{\delta}{1-\delta}\left[s(\hat{a})-\left(\bar{v}^{0}+\sum_{i=1}^{N} v^{i}\left(a(i)^{\prime \prime}\right)\right)\right] \geq \bar{v}^{0}-\min _{k \in \mathcal{N}} v^{0}(a(k)) \\
& \frac{\delta}{1-\delta}\left[s(\hat{a})-\left(\bar{v}^{0}+\sum_{i=1}^{N} v^{i}\left(a(i)^{\prime \prime}\right)\right)\right] \geq \bar{v}^{0}-v^{0}(\hat{a}) .
\end{aligned}
$$


Now assume that without loss of generality $\min _{k \in \mathcal{N}} v^{0}(a(k)) \leq \min _{k \in \mathcal{N}} v^{0}\left(a(k)^{\prime}\right)$ and choose $\ell \in \arg \min _{k \in \mathcal{N}} v^{0}\left(a(k)^{\prime \prime}\right)$ implying that $v^{0}\left(a(\ell)^{\prime \prime}\right)=\min _{k \in \mathcal{N}} v^{0}\left(a(k)^{\prime \prime}\right)$. Notice that, for any $j \in \mathcal{N}$, either $v^{0}\left(a(j)^{\prime \prime}\right)=v^{0}(a(j))$ or $v^{0}\left(a(j)^{\prime \prime}\right)=v^{0}\left(a(j)^{\prime}\right)$ must hold. Then either of the following must also hold;

$$
\min _{k \in \mathcal{N}} v^{0}\left(a(k)^{\prime \prime}\right)=v^{0}\left(a(\ell)^{\prime \prime}\right)=\left\{\begin{array}{l}
v^{0}(a(\ell)) \geq \min _{k \in \mathcal{N}} v^{0}(a(k)) \\
v^{0}\left(a(\ell)^{\prime}\right) \geq \min _{k \in \mathcal{N}} v^{0}\left(a(k)^{\prime}\right) \geq \min _{k \in \mathcal{N}} v^{0}(a(k))
\end{array}\right.
$$

which implies $\min _{k \in \mathcal{N}} v^{0}\left(a(k)^{\prime \prime}\right) \geq \min _{k \in \mathcal{N}} v^{0}(a(k))$. Hence inequality (23) implies that

$$
\frac{\delta}{1-\delta}\left[s(\hat{a})-\left(\bar{v}^{0}+\sum_{i=1}^{N} v^{i}\left(a(i)^{\prime \prime}\right)\right)\right] \geq \bar{v}^{0}-\min _{k \in \mathcal{N}} v^{0}\left(a(k)^{\prime \prime}\right) .
$$

Equation (24) and (25) imply that $\hat{a} \in A_{1}^{P C}\left(\boldsymbol{a}(\mathcal{N})^{\prime \prime}\right)$, or $A_{1}^{P C}\left(\boldsymbol{a}(\mathcal{N})^{\prime \prime}\right) \neq \emptyset$. Thus $\boldsymbol{a}(\mathcal{N})^{\prime \prime} \in A_{0}^{P C}$.

Let

$$
A_{0}^{O P C}:=\left\{\boldsymbol{a}(\mathcal{N}) \in A_{0}^{P C} \mid \nexists \nexists \tilde{\boldsymbol{a}}(\mathcal{N})^{\prime} \in A_{0}^{P C} \text { such that } \begin{array}{l}
{ }^{\forall} j \in \mathcal{N}, v^{j}(\tilde{a}(j)) \leq v^{j}(a(j)) \quad \text { and } \\
{ }^{\exists} k \in \mathcal{N}, v^{k}(\tilde{a}(k))<v^{k}(a(k))
\end{array}\right\},
$$

that is, the Pareto-frontier of $A_{0}^{P C}$. The proof is completed if $A_{0}^{O P C} \neq \emptyset$ and for any $a(\mathcal{N}), a(\mathcal{N})^{\prime} \in$ $A_{0}^{O P C}, v^{j}(a(j))=v^{j}\left(a(j)^{\prime}\right)$ for all $j \in \mathcal{N}$. Since $A_{0}^{P C}$ is nonempty and compact, $A_{0}^{O P C}$ is also nonempty. Furthermore Lemma 8 implies that for any $a(\mathcal{N}), a(\mathcal{N})^{\prime} \in A_{0}^{P C}, a(\mathcal{N})^{\prime \prime} \in A_{0}^{P C}$ and by definition of $\boldsymbol{a}(\mathcal{N})^{\prime \prime} \in A_{0}^{P C}, v^{\ell}\left(a(\ell)^{\prime \prime}\right)=\min \left\{v^{\ell}(a(\ell)), v^{\ell}\left(a(\ell)^{\prime}\right)\right\}$ for all $\ell \in \mathcal{N}$. Then if for some $\boldsymbol{a}(\mathcal{N}), \boldsymbol{a}(\mathcal{N})^{\prime} \in$ $A_{0}^{\text {OPC }}$ there exists $k \in \mathcal{N}$ such that (without loss of generality) $v^{k}(a(k))<v^{k}\left(a(k)^{\prime}\right)$, it is seen that $v^{j}\left(a(k)^{\prime \prime}\right)=\min \left\{v^{k}(a(k)), v^{k}\left(a(k)^{k}\right)\right\}<v^{k}\left(a(k)^{\prime}\right)$, and $v^{\ell}\left(a(\ell)^{\prime \prime}\right) \leq v^{\ell}\left(a(\ell)^{\prime}\right)$ for all $\ell \in \mathcal{N}$. It contradicts the hypothesis that $a(\mathcal{N})^{\prime} \in A_{0}^{O P C}$. Hence $v^{j}(a(j))=v^{j}\left(a(j)^{\prime}\right)$ for all $j \in \mathcal{N}$.

\section{A.7 Proof of Corollary 2}

It is from Lemma 3 and Proposition 4. 


\section{A.8 Proof of Proposition 6}

1. Let $A_{1}^{P C}(\boldsymbol{a}(\mathcal{N}) ; \delta), A_{0}^{P C}(\delta)$, and $\boldsymbol{a}_{0}(\mathcal{N} ; \delta) \equiv\left(a_{0}(1 ; \delta), \ldots, a_{0}(N ; \delta)\right)$ be the redefinition of $A_{1}^{P C}(\boldsymbol{a}(\mathcal{N}))$, $A_{0}^{P C}$, and $\boldsymbol{a}_{0}(\mathcal{N})$, respectively for expressing $\delta$ explicitly. We will show that if $1>\delta^{\prime}>\delta \geq 0$, then $v^{j}\left(a_{0}\left(j ; \delta^{\prime}\right)\right) \leq v^{j}\left(a_{0}(j ; \delta)\right)$ for all $j \in \mathcal{N}$. It is easy to see that if $\hat{a} \in A_{1}^{P C}(\boldsymbol{a}(\mathcal{N}) ; \delta)$, then $\hat{a} \in A_{1}^{P C}\left(\boldsymbol{a}(\mathcal{N}) ; \delta^{\prime}\right)$ which means that $A_{1}^{P C}(\boldsymbol{a}(\mathcal{N}) ; \delta) \subset A_{1}^{P C}\left(\boldsymbol{a}(\mathcal{N}) ; \delta^{\prime}\right)$ for any given $\boldsymbol{a}(\mathcal{N}) \in \mathcal{A}^{N}$. It implies that if $A_{1}^{P C}(\boldsymbol{a}(\mathcal{N}) ; \delta) \neq \emptyset$, then $A_{1}^{P C}\left(\boldsymbol{a}(\mathcal{N}) ; \delta^{\prime}\right) \neq \emptyset$. Then by definition, we obtain $A_{0}^{P C}(\delta) \subset A_{0}^{P C}\left(\delta^{\prime}\right)$. Then because of Proposition 4 , we see that $a_{0}(\mathcal{N} ; \delta) \in A_{0}^{P C}\left(\delta^{\prime}\right)$. Furthermore Proposition 4 tells us that for all $j \in \mathcal{N}, v^{j}\left(a_{0}\left(j ; \delta^{\prime}\right)\right) \leq v^{j}(a(j))$ for all $a(\mathcal{N}) \in A_{0}^{P C}\left(\delta^{\prime}\right)$. Therefore we obtain the desired result.

2. If there exists $\hat{a} \in \mathcal{A}$ such that $s(\hat{a})-\left(\bar{v}^{0}+\sum_{i=1}^{N} \underline{v}^{i}\right)>0$, the left-hand side of equation (16) is unbounded above in $\delta \in[0,1)$. Then we can pick $\bar{\delta}^{O P C *} \in[0,1)$ which satisfies equation (16).

Thus conversely suppose that $s(\hat{a})-\left(\bar{v}^{0}+\sum_{i=1}^{N} \underline{v}^{i}\right) \leq 0$ for all $\hat{a} \in \mathcal{A}$. However, whenever we choose $\bar{a}^{0} \in \bar{A}^{0}, s\left(\bar{a}^{0}\right)=\sum_{j=0}^{N} v^{j}\left(\bar{a}^{0}\right)=\bar{v}^{0}+\sum_{j=1}^{N} v^{j}\left(\bar{a}^{0}\right) \geq \bar{v}^{0}+\sum_{j=1}^{N} \underline{v}^{j}$, which implies that $s\left(\bar{a}^{0}\right)-$ $\left(\bar{v}^{0}+\sum_{j=1}^{N} \underline{v}^{j}\right) \geq 0$. Thus we can find a decision $\bar{a}^{0}$ which satisfies $s\left(\bar{a}^{0}\right)-\left(\bar{v}^{0}+\sum_{j=1}^{N} \underline{v}^{j}\right)=0$. Furthermore Since $s\left(\bar{a}^{0}\right)=\bar{v}^{0}+\sum_{j=1}^{N} v^{j}\left(\bar{a}^{0}\right)$, we immediately obtain $\sum_{j=1}^{N} v^{j}\left(\bar{a}^{0}\right)=\sum_{j=1}^{N} \underline{v}^{j}$. Because $v^{j}\left(\bar{a}^{0}\right) \geq \underline{v}^{j}$ for all $j \in \mathcal{N}, v^{j}\left(\bar{a}^{0}\right)=\underline{v}^{j}$ for all $j \in \mathcal{N}$ implying that $\bar{a}^{0} \in \underline{A}^{j}$ for all $j \in \mathcal{N}$. Then we see that a vector $\left(\bar{a}^{0}, \bar{a}^{0}, \ldots, \bar{a}^{0}\right) \in \mathcal{A} \times \prod_{j=1}^{N} \underline{A}^{j}$ satisfies inequality equation (16) holds (because both sides are 0). From Corollary 2, the result is established.

\section{A.9 Proof of Corollary 3}

When $\delta>0$, the proof is as in the text. Suppose that $\delta=0$ and $\hat{\Sigma}^{*}(\hat{a}) \neq \emptyset$. It means that there exists a decision stationary SPE of $\hat{a}$ by which the payoff of principal $j$ is at most $v^{j}(\hat{a})$. Because it is less than $v^{j}\left(a_{0}(j)\right)$ by hypothesis, from Lemma 3 it contradicts that $v^{j}\left(a_{0}(j)\right)$ is the minimum SPE payoff for principal $j$. 


\section{A.10 Proof of Proposition 8}

(Necessity) Suppose that $\hat{w}^{j}(\hat{a})>\delta\left[v^{j}(\hat{a})-v^{j}\left(a_{0}(j)\right)\right]$ for some $j \in \mathcal{N}$. Then the SMA-equilibrium payoff of principal $j$ is $\hat{y}^{j}=v^{j}(\hat{a})-\hat{w}^{j}(\hat{a})<(1-\delta) v^{j}(\hat{a})+\delta v^{j}\left(a_{0}(j)\right)$. It cannot be attained in any RPC-equilibria from Proposition 7.

(Sufficiency) If $\left(\hat{a}, \hat{w}^{1}(\cdot), \ldots, \hat{w}^{N}(\cdot)\right)$ is a SMA-equilibrium, the net payoff vector $\left(\hat{y}^{0}, \hat{y}^{1}, \ldots, \hat{y}^{N}\right)$ must be larger than or equal to the minimax payoff and $\hat{y}^{j}$ is less than or equal to $v^{j}(\hat{a})$ for each $j \in \mathcal{N}$ because the payment must be nonnegative. Then if $\left(\hat{y}^{0}, \hat{y}^{1}, \ldots, \hat{y}^{N}\right)$ is an SMA-equilibrium net payoff vector, it satisfies

$$
\left(\hat{y}^{0}, \hat{y}^{1}, \ldots, \hat{y}^{N}\right) \in Y(\hat{a}):=\left\{\left(y^{0}, y^{1}, \ldots, y^{N}\right) \mid \sum_{j=0}^{N} y^{j}=s(\hat{a}), y^{0} \geq \bar{v}^{0}, v^{j}(\hat{a}) \geq y^{j} \geq \underline{v}^{j},{ }^{\forall} j \in \mathcal{N}\right\} .
$$

Now suppose that $\hat{w}^{j}(\hat{a}) \leq \delta\left[v^{j}(\hat{a})-v^{j}\left(a_{0}(j)\right)\right]$ for all $j \in \mathcal{N}$. Then it is easy to see that $Y(\hat{a})$ is a subset of $\hat{U}^{*}(\hat{a})$ which implies that the payoff vector $\left(\hat{y}^{0}, \hat{y}^{1}, \ldots, \hat{y}^{N}\right) \in \hat{U}^{*}(\hat{a})$ is also a RPC-equilibrium payoff vector.

\section{A.11 Proof of Proposition 9}

(Necessity) Suppose that principal $j$ satisfying equation (18). The SMA-equilibrium payoff of principal $j$ is $v^{j}(\hat{a})-\hat{w}^{j}(\hat{a})=\underline{v}^{j}$. Notice that $v^{j}\left(a_{0}(j)\right) \geq \underline{v}^{j}$ for any $\delta \in[0,1)$ and then $(1-\delta) v^{j}(\hat{a})+$ $\delta v^{j}\left(a_{0}(j)\right) \geq(1-\delta) v^{j}(\hat{a})+\delta \underline{v}^{j}>\underline{v}^{j}$ for any $\delta \in[0,1)$. Then from Proposition 7 , it is obvious that it cannot be attained for any $\delta \in[0,1)$ by RPC-equilibria.

(Sufficiency) First of all, recall that, from Proposition 6 , there exists $\bar{\delta}^{O P C *} \in[0,1)$ such that for $\delta \in\left[\bar{\delta}^{\mathrm{OPC} *}, 1\right), u^{j}\left(a_{0}(j)\right)=\underline{v}^{j}$. Then for $\delta \in\left[\bar{\delta}^{\mathrm{OPC} *}, 1\right)$,

$$
\hat{U}^{*}(\hat{a})=\left\{\left(u^{0}, u^{1}, \ldots, u^{N}\right) \mid \sum_{j=0}^{N} u^{j}=s(\hat{a}), u^{0} \geq \bar{v}^{0}, v^{j}(\hat{a}) \geq u^{j} \geq(1-\delta) v^{j}(\hat{a})+\delta \underline{v}^{j},{ }^{\prime} j \in \mathcal{N}\right\} .
$$

Now suppose that every principal violates equation (18), which means that for every $j \in \mathcal{N}$, if 
$v^{j}(\hat{a})>\underline{v}^{j}$, then $\hat{w}^{j}(\hat{a}) \neq v^{j}(\hat{a})-\underline{v}^{j}$. Let $\tilde{J}(1):=\left\{j \in \mathcal{N} \mid v^{j}(\hat{a})>\underline{v}^{j}\right\}$ and $\tilde{J}(2):=\mathcal{N} \backslash \tilde{J}(1)$. Since $v^{j}(\hat{a})=\underline{v}^{j}$ for $j \in \tilde{J}(2)$, for $\delta \in\left[\bar{\delta}^{O P C *}\right.$,1) $\hat{U}^{*}(\hat{a})$ can be written as

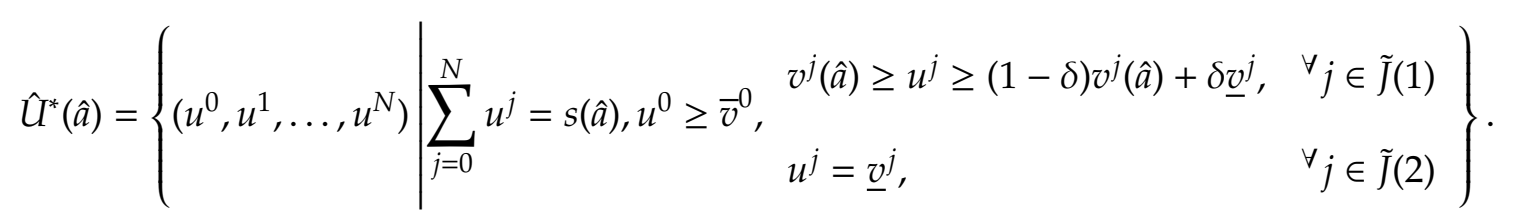

Note that the SMA-equilibrium net payoff is $\hat{y}^{j}=v^{j}(\hat{a})-\hat{w}^{j}(\hat{a})$ for $j \in \tilde{J}(1)$ and $\hat{y}^{j}=\underline{v}^{j}$ for $j \in \tilde{J}(2)$. Then the SMA-equilibrium net payoff vector is in $\hat{U}^{*}(\hat{a})$ if $\hat{y}^{j} \in\left[(1-\delta) v^{j}(\hat{a})+\delta \underline{v}^{j}, v^{j}(\hat{a})\right]$ for all $j \in \tilde{J}(1)$ for some $\delta$. Let $\tilde{\delta}:=\max \left\{\bar{\delta}^{O P C *}, \max _{j \in \tilde{J}(1)} \hat{w}^{j}(\hat{a}) /\left(v^{j}(\hat{a})-\underline{v}^{j}\right)\right\}$. Since the SMA-equilibrium payoff is no less than the minimax value and $\hat{w}^{j}(\hat{a}) \neq v^{j}(\hat{a})-\hat{w}^{j}(\hat{a})$ for for each $j \in \tilde{J}(1)$, we obtain $v^{j}(\hat{a})-\hat{w}^{j}(\hat{a})>\underline{v}^{j}$ which implies that $\tilde{\delta}<1$. Furthermore it is easy to see that when $\delta \geq \tilde{\delta}$, then $\hat{y}^{j} \in\left[(1-\delta) v^{j}(\hat{a})+\delta \underline{v}^{j}, \bar{v}^{j}\right]$ for $j \in \tilde{J}(1)$. Therefore it is shown that the SMA-equilibrium payoff vector is in $\hat{U}^{*}(\hat{a})$ for sufficiently high $\delta$.

\section{A.12 Proof of Lemma 6}

For any subset of principals $J \subset \mathcal{N}$, let $M^{j}:=s^{*}-\max _{a \in \mathcal{F}}\left[s(a)-\sum_{j \in J} v^{j}(a)\right]$ which is called the marginal contribution of a subset of principals $J$. Let $\left(\hat{a}, \hat{w}^{1}(\cdot), \ldots, \hat{w}^{N}(\cdot)\right)$ be a SMAT-equilibrium and $\left(\hat{y}^{0}, \hat{y}^{1}, \ldots, \hat{y}^{N}\right)$ be the corresponding net payoff. Theorem 1 of Bergemann and Välimäki (2003) implies a useful result to show this statement.

Lemma 9 A vector $\left(\hat{y}^{0}, \hat{y}^{1}, \ldots, \hat{y}^{N}\right)$ is one of net payoff vectors on SMAT-equilibria only if for all subsets of the principals $S \subset \mathcal{N}, \sum_{i \in S} \hat{y}^{i} \leq M^{S}$.

Proof (Lemma 9) This is the weaker condition of Theorem 1 of Bergemann and Välimäki (2003) which provides the necessary and sufficient condition for SMAT-equilibrium payoff.

Suppose that $\underline{A}^{j} \cap A^{*} \neq \emptyset$ and then we can choose an element $\underline{a^{j}} \in \underline{A}^{j} \cap A^{*}$. Then such $\underline{a}^{j}$ satisfies $s\left(\underline{a}^{j}\right)=\max _{a \in \mathcal{A}} s(a)=s^{*}$ and $v^{j}\left(\underline{a}^{j}\right)=\min _{a \in \mathcal{A}} v^{j}(a)=\underline{v}^{j}$ and notice that

$$
s\left(\underline{a}^{j}\right)-v^{j}\left(\underline{a}^{j}\right) \leq \max _{a \in \mathcal{A}}\left[s(a)-v^{j}(a)\right] \leq \max _{a \in \mathcal{A}} s(a)-\min _{a \in \mathcal{A}} v^{j}(a)=s^{*}-\underline{v}^{j}=s\left(\underline{a}^{j}\right)-v^{j}\left(\underline{a}^{j}\right)
$$


which implies that $\max _{a \in \mathcal{H}}\left[s(a)-v^{j}(a)\right]=s\left(\underline{a}^{j}\right)-v^{j}\left(\underline{a}^{j}\right)=s^{*}-\underline{v}^{j}$. Then by applying Lemma 9 for a singleton set of principal $j$, we obtain that

$$
\hat{y}^{j} \leq s^{*}-\left[s^{*}-\underline{v}^{j}\right]
$$

or equivalently $\hat{y}^{j} \leq \underline{v}^{j}$. However since the equilibrium net payoff must not be less than the minimax payoff which is $\underline{v}^{j}$ for principal $j$, it follows that $\hat{y}^{j}=\underline{v}^{j}$. Notice that $\hat{y}^{j}=v^{j}(\hat{a})-\hat{w}^{j}(\hat{a})$, meaning that $\hat{w}^{j}(\hat{a})=v^{j}(\hat{a})-\underline{v}^{j}$.

\section{A.13 Proof of Proposition 11}

Since $s(a)=s^{*}$ for all $a \in \mathcal{A}$, it is straightforward that $A^{*}=\mathcal{A}$ immediately implying that $\underline{A}^{i} \cap$ $A^{*} \neq \emptyset$ for all $i \in \mathcal{N}$. Then Lemma 6 implies that for any SMAT-equilibrium $\left(\hat{a}, \hat{w}^{1}(\cdot), \ldots, \hat{w}^{N}(\cdot)\right)$, $\hat{w}^{i}(a)=v^{i}(a)-\underline{v}^{i}$ for all $a \in \mathcal{A}$ and $i \in \mathcal{N}$. Then thanks to Proposition 9, if the decision on the SMAT-equilibrium satisfies $v^{j}(\hat{a})>\underline{v}^{j}$ for some $j \in \mathcal{N}$, such a SMAT-equilibrium payoff cannot be supported on RPC-equilibria. Then conversely suppose that $v^{j}(\hat{a})=\underline{v}^{j}$ for all $j \in \mathcal{N}$. Now by our hypothesis $\hat{a} \notin \bar{A}^{0}$ which means that there exists $\bar{a}^{0} \in \bar{A}^{0}$, or equivalently $v^{0}\left(\bar{a}^{0}\right)>v^{0}(\hat{a})$. Since $s(\hat{a})=s\left(\bar{a}^{0}\right)\left(=s^{*}\right)$, it implies that

$$
\sum_{i \in \mathcal{N}} v^{i}\left(\bar{a}^{0}\right)<\sum_{i \in \mathcal{N}} v^{i}(\hat{a})=\sum_{i \in \mathcal{N}} \underline{v}^{i}
$$

which is a contradiction because it is obvious that $v^{i}\left(\bar{a}^{0}\right) \geq \underline{v}^{i}$ for all $i \in \mathcal{A}$.

\section{References}

Abreu, D. (1986): “Extremal Equilibria of Oligopolistic Supergame," Journal of Economic Theory, 39, $191-225$.

(1988): “On the Theory of Infinitely Repeated Games with Discounting," Econometrica, 56, 
383-396.

Aragonès, E., T. Palfrey, and A. Postlewaite (2007): "Political Reputations and Campaign Promises," Journal of European Economic Association, 5, 846-884.

Baker, G., R. Gibbons, and K. J. Murphy (2002): "Relational Contracts and the Theory of the Firm," Quarterly Journal of Economics, 117, 39-84.

Bergemann, D. and J. Välimäki (2003): “Dynamic Common Agency,” Journal of Economic Theory, $111,23-48$.

Bernheim, B. D. and M. D. Whinston (1986a): “Common Agency,” Econometrica, 54, 923-942. (1986b): “Menu Auctions, Resource Allocation, and Economic Infuluence," Quarterly Journal of Economics, 101, 1-32.

Besley, T. And S. Coate (2001): “Lobbying and Welfare in a Representative Democracy," Review of Economic Studies, 68, 67-82.

Campante, F. R. and F. H. Ferreira (2007): “Inefficient Lobbying, Populism and Oligarchy,” Journal of Public Economics, 91, 993-1021.

Dixit, A., G. M. Grossman, and E. Helpman (1997): “Common Agency and Coordination: General Theory and Applicatoin to Government Policy Making," Journal of Political Economy, 105, 752769.

FonG, Y.-F. AND J. Li (2008): “Relational Contract, Limited Liability, and Employment Dynamics," mimeo, Northwestern Univesity.

Fredriksson, P. G. (1997): “The Political Economy of Pollution Taxes in a Small Open Economy," Journal of Environmental Economics and Management, 33, 44-58.

Fudenberg, D. ANd E. MAskin (1986): “The Folk Theorem in Repeated Games with Discounting or with Incomplete Information," Econometrica, 54, 533-554. 
Grossman, G. M. and E. Helpman (1994): "Protection for Sale," American Economic Review, 84, 833-850.

(1996): "Electoral Competition and Special Interest Politics," Review of Economic Studies, 63, 265-286.

(2001): Special Interest Politics, Cambridge, MA: MIT Press.

Kvaløy, O. and T. E. Olsen (2006): “Team Incentives in Relational Employment Contracts," Journal of Labor Economics, 24, 139-169.

Levin, J. (2002): “Multilateral Contracting and the Employment Relationship," Quarterly Journal of Economics, 117, 1075-1104.

_ (2003): “Relational Incentive Contracts," American Economic Review, 93, 835-857.

MacLeod, W. B. and J. M. Malcomson (1989): “Implicit Contracts, Incentive Compatibility, and Involuntary Unemployment," Econometrica, 57, 447-480.

Martimort, D. (2006): "Multi-Contracting Mechanism Design," in Advances in Economics and Econometrics, Theory and Applications: Ninth World Congress of the Econometric Society Volume 1, ed. by R. Blundell, W. K. Newey, and T. Persson, New York: Cambridge University Press, 57-101.

Martimort, D. and L. Stole (2009): “Market Participation in Delegated and Intrinsic CommonAgency Games," RAND Journal of Economics, 40, 78 - 102.

Maskin, E. And J. Tirole (2001): “Markov Perfect Equilibrium I. Observable Actions,” Journal of Economic Theory, 100, 191-219.

McCarty, N. and L. S. Rothenberg (1996): “Commitment and the Campaign Contribution Contract," American Journal of Political Science, 40, 872-904.

Prat, A. And A. Rustichini (2003): “Games Played through Agents," Econometrica, 71, 989-1026. 
Rayo, L. (2007): "Relational Incentives and Moral Hazard in Teams," Review of Economic Studies, 74, 937-963.

SNYder, JR., J. M. (1992): “Long-term Investing in Politicians; Or, Give Early, Give Often,” Journal of Law and Economics, 35, 15-43. 